Bundesgesundheitsbl 2015 · 58:889-907 DOI 10.1007/s00103-015-2193-7

Online publiziert: 17. Juli 2015

๑) Springer-Verlag Berlin Heidelberg 2015
Bekanntmachung des Umweltbundesamtes

\section{Stoffmonographie für 1,2,5,6,9, 10-Hexabromcyclododecan (HBCDD) - HBM-Werte für HBCDD im Fettanteil der Muttermilch oder des Blutplasmas}

\section{Stellungnahme der Kommission „Human- Biomonitoring" des Umweltbundesamtes}

\begin{tabular}{|ll}
\hline Abkürzungen \\
\hline$A G S$ & Ausschuss für Gefahrstoffe \\
\hline BAEP & „brainstem auditory evoked potentials“ akustisch evozierte Hirnstammpotentiale \\
\hline$B M D$ & Benchmark Dose \\
\hline$B M D L$ & Benchmark Dose Lower Limit \\
\hline CYP2B & Cytochrom P450 Familie 2, Subfamilie B \\
\hline CYP3A & Cytochrom P450 Familie 3, Subfamilie A \\
\hline$D M S O$ & Dimethylsulfoxid \\
\hline$D N E L$ & Derived No Effect Level \\
\hline$E C H A$ & European Chemicals Agency \\
\hline$E F S A$ & European Food Safety Authority \\
\hline$E U$ & European Union - Europäische Union \\
\hline GLP & Good Laboratory Practise \\
\hline$H B C D D$ & oder auch HBCD, Hexabromcyclododecan \\
\hline IOM & Institute of Occupational Medicine \\
\hline KG & Körpergewicht \\
\hline LLNA & Local Lymph Node Assay \\
\hline LOAEL & Lowest Observed Adverse Effect Level \\
\hline MoE & Margin of Exposure \\
\hline$N A D P H$ & reduzierte Form des Nicotinsäureamid-Adenin-Dinukleotid-Phosphat \\
\hline NICNAS & National Industrial Chemicals Notification and Assessment Scheme \\
\hline NOAEL & No Observed Adverse Effect Level \\
\hline$O E C D$ & Organisation for Economic Co-operation and Development \\
\hline$P N D / P N W$ & Postnatal Day/Postnatal Week \\
\hline$P O D$ & Point Of Departure \\
\hline$P O P$ & Persistent Organic Pollutant \\
\hline SVHC & Substances of Very High Concern - besonders besorgniserregende Stoffe \\
\hline$T 3$ & Trijodthyronin \\
\hline$T 4$ & Thyronin \\
\hline$T D I$ & Tolerable Daily Intake \\
\hline$T S H$ & Thyronin stimulierendes Hormon \\
\hline
\end{tabular}

\section{Einleitung}

Für die Substanz Hexabromcyclododecan (HBCDD, CAS-Nr. 25637-99-4, EINECSNr. 247-148-4) liegen umfangreiche Daten zur Toxikologie vor. Die oben genannte Cas-Nr. bezieht sich auf HBCDD ohne Spezifikation der Position der Bromatome. Für 1,2,5,6,9,10-Hexabromcyclododecan gilt die CAS-Nr. 3194-55-6 (EINECS-Nr. 221-695-9). Nach EU Altstoffbewertung [1] enthält das technische HBCDD hauptsächlich 3 chirale Diastereomere (alpha-, CAS-Nr. 134237-50-6; beta-, CAS-Nr. 134237-51-7 und gamma-HBCDD, CASNr. 134237-52-8). Der Gehalt ist abhängig von den Herstellern und der jeweils angewendeten Produktionsweise, und variiert im Bereich von 70-95\% gamma-HBCDD sowie $3-30 \%$ alpha-HBCDD und betaHBCDD [1-3]. Darüber hinaus wurden in technischem HBCDD auch noch 2 weitere Isomere in geringeren Mengen gefunden: delta-HBCDD mit 0,5\% und epsilonHBCDD mit 0,3\% [4].

Die ausführlichste Darstellung aller toxikologisch relevanten Studien bis zum Jahr 2008 findet sich in der Altstoffbewertung der EU [1]. Zusätzliche Studien bis zum Jahr 2010 sind in den Begründungen der Europäischen Chemikalienagentur ECHA für eine harmonisierte Einstufung [5] sowie für die Aufnahme in die Liste der besonders besorgniserregenden Stoffe 


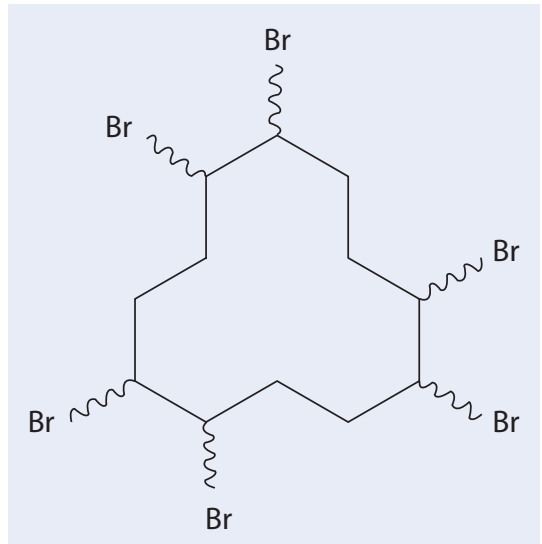

Abb. $1 \Delta$ Strukturformel von HBCDD [1]

(substances of very high concern, SVHC) [6] enthalten. Die Europäische Behörde für Lebensmittelsicherheit (EFSA) bewertete das Vorkommen von HBCDD in Lebensmitteln [7]. Weiterhin wurde im Jahr 2012 eine Bewertung in Australien abgeschlossen, die alle Studien bis 2011 enthält [3].

HBCDD wurde auch im Altstoffprogramm der Organisation für wirtschaftliche Zusammenarbeit und Entwicklung (OECD) [8] behandelt. Eine weitere Prüfung im Hinblick auf krebserzeugende, erbgutverändernde und fortpflanzungsgefährdende Eigenschaften wurde vom Ausschuss für Gefahrstoffe vorgenommen [9].

Die Eigenschaften von HBCDD wurden auch im Zusammenhang mit der Einstufung als persistenter organischer Schadstoff untersucht [10-13]. Der Stoff wurde im Mai 2013 in die Stockholmer Konvention über persistente organische Schadstoffe, POPs, aufgenommen [14, 15]. Für den Einsatz als Flammschutzmittel gilt damit nach einer Übergangsfrist ein weltweites Verwendungs-und Handelsverbot. Unter REACH ist die uneingeschränkte Herstellung und Verwendung von HBCDD in der EU nur noch bis zum 21. August 2015 möglich. Inwiefern danach befristete Zulassungen für spezielle Einsatzgebiete von HBCDD unter Inanspruchnahme von Ausnahmeregeln nach der Stockholmer Konvention möglich sind, ist derzeit noch offen [16].

\section{Physiko-chemische Eigenschaften}

Bei Raumtemperatur liegt technisches HBCDD als weißer Feststoff vor und hat keinen wahrnehmbaren Geruch. Die molare Masse beträgt $641 \mathrm{~g} / \mathrm{mol}$.

Die Strukturformel von HBCDD ist in

- Abb. 1 dargestellt. Die stereochemische Ausrichtung der Bromatome ist nicht differenziert.

Weitere physiko-chemische Eigenschaften sind in der $\bullet$ Tab. 1 dargestellt.

\section{Verwendung und Exposition}

HBCDD wird hauptsächlich als Flammschutzmittel in expandiertem PolystyrolSchaumstoff - EPS (expanded polystyrene foam) und extrudiertem PolystyrolHartschaum - XPS (extruded polystyrene foam) für die Herstellung von Wärmedämmplatten verwendet $[17,18]$. In diesen beiden Bereichen werden innerhalb der EU je 5300 und 5900 t HBCDD pro Jahr eingesetzt. Geringere Mengen (ca. 200 t/a) werden in mikronisierter Form in Textilen eingesetzt [19]. Insgesamt wird die Verbrauchsmenge innerhalb der EU für den Zeitraum 2006/2007 auf 11.600 t/a geschätzt [19]. In der EU produziert wurden im Jahr 2006 lediglich 6000 t.

\subsection{Vorkommen in der Luft}

Studien zum Vorkommen von HBCDD am Arbeitsplatz sind in den Dokumenten der EFSA [7] und der EU [1] dargestellt. Bei der Herstellung von HBCDD werden Werte von 9400 bis $28.500 \mathrm{ng} / \mathrm{m}^{3}$ Luft berichtet. Höhere Werte von $<13$ bis $1600 \mu \mathrm{g} / \mathrm{m}^{3}$ Luft wurden bei der Produktion von expandiertem Polystyrol ermittelt. Eine Übersicht der gemessenen Konzentrationen von HBCDD in der Innenraumluft von Wohnräumen und Büros findet sich in $\bullet$ Tab. 2. Hier liegen die Maximalwerte bei $1,3 \mathrm{ng} / \mathrm{m}^{3}$. Auch in der Innenraumluft von Fahrzeugen kann HBCDD nachgewiesen werden [20].

Eine Übersicht der gemessenen Konzentrationen von HBCDD in Hausstaub ist in - Tab. 3 dargestellt. Die Werte beziehen sich soweit nicht anders angegeben auf Gesamt-HBCDD. Der Median der Werte in Europa liegt im Bereich von 45 bis 3250 ng/g. Maximalwerte in Großbritannien reichen bis $140.000 \mathrm{ng} / \mathrm{g}$. Die Werte aus anderen Teilen der Welt liegen in der gleichen Größenordnung. Es wurde in einzelnen Arbeiten auch nach Isome- ren unterschieden, diese Ergebnisse sind in der Tabelle jedoch nicht dargestellt. Bei den Untersuchungen in Tschechien [24] stellte gamma-HBCDD mit $69 \%$ das dominierende Isomer dar (alpha-HBCDD $25 \%$, beta-HBCDD 6\%). Demgegenüber war in Belgien alpha-HBCDD (59\%, 72\%) dominierend (gamma-HBCDD $29 \%$, $15 \%$, beta-HBCDD $12 \%, 13 \%)$. Dieser erhöhte Anteil von alpha-HBCDD im Hausstaub kann nach Roosens et al. [25] damit zusammenhängen, dass unter Einfluss von Sonnenlicht eine Isomerisierung von gamma- zu alpha-HBCDD erfolgt [26]. Weiterhin wurde in Textilien ein erhöhter Anteil von alpha-HBCDD gefunden. Dieser erhöhte Anteil wird auf die Isomerisierung während des Erhitzens, durch das HBCDD in das Material eingebracht wird, oder auf die bevorzugte Adsorption des alpha-HBCDD an die Textiloberfläche zurückgeführt [27].

Neben den Isomeren können auch Abbauprodukte im Hausstaub nachgewiesen werden $[22,28]$. Hierbei handelt es sich um tetra- und penta-bromierte Substanzen (Tetrabromcyclododecane (TBCDDe), Pentabromcyclododecane (PBCDDe)). Diese wurden jedoch nicht quantifiziert.

\subsection{Vorkommen in der Muttermilch}

In - Tab. 4 sind die Ergebnisse der Untersuchungen von Muttermilch zusammengefasst. Der Median der Werte in Europa liegt im Bereich von 0,13 bis 3,9 ng/g Fett (Maximalwerte bis 22,4 ng/g Fett). Die Werte aus anderen Teilen der Welt liegen in der gleichen Größenordnung. Eine Ausnahme bildet eine Untersuchung in Spanien aus den Jahren 2006-2007 mit Konzentrationen im Bereich von 3 bis 188 ng/g Fett. Nach Angaben der EFSA [7] wurden in 2009 geringere Werte (max. $6,2 \mathrm{ng} / \mathrm{g}$ Fett) gemessen.

Zeitreihen sind für gepoolte Proben aus Schweden verfügbar. Hier zeigt sich von 1980 (<0,1 ng/g Fett) bis 2004 (0,4 ng/g Fett) ein Anstieg der Konzentrationen in Muttermilchproben aus Stockholm [42]. Im Zeitraum von 1996-2006 lagen $>60 \%$ der Proben $(n=335)$ aus Uppsala unterhalb der Bestimmungsgrenze (LOQ) von 0,2 ng/g Fett [43]. EFSA [7] berichtet für Stockholm Werte von 0,62 
Bundesgesundheitsbl 2015 ·58:889-907 DOI 10.1007/s00103-015-2193-7

(c) Springer-Verlag Berlin Heidelberg 2015

Bekanntmachung des Umweltbundesamtes

\section{Stoffmonographie für 1,2,5,6,9,10-Hexabromcyclododecan (HBCDD) - HBM-Werte für HBCDD im Fettanteil der Muttermilch oder des Blutplasmas. Stellungnahme der Kommission „Human-Biomonitoring“ des Umweltbundesamtes}

\section{Zusammenfassung}

Hexabromcyclododecan (HBCDD) wird hauptsächlich als Flammschutzmittel in Wärmedämmplatten und Textilien eingesetzt. Für die Substanz liegen umfangreiche Daten zum Vorkommen in Hausstaubproben, Muttermilch, menschlichem Blut und Fettgewebe vor. Die EFSA (European Food Safety Authority) identifizierte Effekte auf die Entwicklung des Nervensystems und das Verhalten als kritischen Endpunkt toxikologischer Untersuchungen und leitete hierfür eine $\mathrm{BMDL}_{10}$ von $0,93 \mathrm{mg} / \mathrm{kg} \mathrm{KG}$ ab. In Übereinstimmung mit dem EFSA Panel on Contaminants in the Food Chain (European Food Safety Authority (EFSA), EFSA Journal 9(7):2296, 2011) erachtet auch die HBM-Kommission die Studie von Eriksson et al. (Eriksson et al., Environ Toxicol Pharmacol 21(3):317-322, 2006) als Schlüs- selstudie und verwendet die hieraus resultierende $\mathrm{BMDL}_{10}$ von $0,93 \mathrm{mg} / \mathrm{kg} \mathrm{KG}$ als Ausgangspunkt (point of departure - POD) für die Ableitung eines HBM-Wertes. Die zur Verfügung stehenden toxikokinetischen Daten zeigen, dass oral verabreichtes HBCDD leicht resorbiert wird und sich im Fettgewebe anreichert. Da sich die Eliminationskinetik für Nagetiere von der des Menschen unterscheidet, können die mit Effekten assoziierten äuBeren Dosen aus dem Tierversuch nicht direkt für die Risikobewertung beim Menschen herangezogen werden. Vielmehr stellt die innere Dosis oder Körperlast das geeignete Maß für einen direkten Vergleich der Effekte auf Tier und Mensch dar. Aus der BMDL 10 von $0,93 \mathrm{mg} / \mathrm{kg}$ KG nach einmaliger oraler Gabe leitet sich unter Berücksichtigung einer oralen Resorption bei Nagetieren von 85\% eine tolerable Körperlast von $0,79 \mathrm{mg} / \mathrm{kg} \mathrm{KG}$ ab. Dieser Wert muss durch einen Faktor von 0,32 dividiert werden (Körperfettgehalt bei Frauen $32 \%$ ), um die tolerable innere Dosis auf das Lipid-Kompartiment zu beziehen. Nach Beaufschlagung mit einem GesamtAssessment-Faktor von 8 (Toxikodynamik: Interspezies $=2,5$ und Intraspezies $=\sqrt{10}$ ) wird für HBCDD ein HBM-I-Wert von gerundet $0,3 \mu \mathrm{g} / \mathrm{g}$ Fett $(1,6 \mu \mathrm{g} / \mathrm{l}$ Plasma) festgelegt. Die HBCDD Konzentrationen im Fettgewebe der Allgemeinbevölkerung liegen zwischen $<0,08$ und $10 \mathrm{ng} / \mathrm{g}$ Fett (Median) und damit deutlich unter dem HBM-I-Wert.

\section{Substance monograph for 1,2,5,6,9,10-hexabromocyclododecane (HBCDD) - HBM levels for HBCDD in the fatty component of breast milk or of blood plasma. Statement of the Human Biomonitoring Committee of the German Federal Environment Agency}

\begin{abstract}
Hexabromocyclododecane (HBCDD) is mainly used as flame retardant in thermal insulation foams and in textile coatings. A comprehensive amount of data on the occurrence of HBCDD in house dust, mother milk, human blood and fatty tissue is available. EFSA (European Food Safety Authority) identified neurodevelopmental effects on behaviour as the critical endpoint of toxicological studies, and derived a benchmark dose lower confidence limit for a benchmark response of 10\% (BM$\mathrm{DL}_{10}$ ) of $0.93 \mathrm{mg} / \mathrm{kg}$ body weight. In accordance with the EFSA Panel on Contaminants in the Food Chain (European Food Safety Authority (EFSA), EFSA Journal 9(7):2296, 2011) the HBM Commission considers the study of Eriksson et al. (Eriksson et al., Environ Toxicol
\end{abstract}

Pharmacol 21(3):317-322, 2006) as key study and the resulting $B M D L_{10}$ as point of departure (POD) for the derivation of the HBM value. The available toxicokinetic data suggest that orally administered HBCDD is easily absorbed and accumulates in lipid tissue. Because elimination kinetics of HBCDD in rodents and humans differ, external dose levels of HBCDD associated with toxic effects in animals cannot be simply extrapolated for the risk assessment in humans. Instead, the internal dose or body burden provides a more appropriate dose metric for a direct comparison of effects in animals and humans. Based on the calculated BMDL 10 value of $0.93 \mathrm{mg} / \mathrm{kg}$ bw as derived from the Eriksson et al. study using a single oral administration, and on the consideration of an oral absorption in rodents of $85 \%$, a body burden at the BMDL 10 of $0.79 \mathrm{mg} / \mathrm{kg}$ bw was derived. With reference to a proportion of $32 \%$ fatty tissue (women) the value of $0.79 \mathrm{mg} / \mathrm{kg}$ bw has to be divided by a factor of 0,32 to relate the tolerable internal dose to the lipid compartment of the body. With standard assessment factors (AFs) for differences in toxicodynamics (interspecies factor $=2.5$, intraspecies factor $=\sqrt{10}$ and total assessment factor $(\mathrm{TAF})=8$ ) the $\mathrm{HBM}$-I value was finally set at $0,3 \mu \mathrm{g} / \mathrm{g}$ lipid $(1,6 \mu \mathrm{g} / \mathrm{l}$ Plasma). HBCDD concentrations in adipose tissue of the general population range from $<0,08$ to $10 \mathrm{ng} / \mathrm{g}$ fat (median), which is clearly below the HBM-I value. und $0,8 \mathrm{ng} / \mathrm{g}$ Fett für die Jahre 2009 und 2010, die den ansteigenden Trend bestätigen (zitiert nach [44], hier jedoch ohne Beleg). Ähnliche Werte zeigen sich auch in Japan $[45,46]$. Die Werte lagen zwischen 1973 und 1983 unterhalb der Nachweisgrenze von 0,1 ng/g Fett. Im Jahr 1988 wurden $0,43 \mathrm{ng} / \mathrm{g}$ Fett nachgewiesen. Zwischen 1993 und 2009 streuten die Werte zwischen 0,62 und 1,9 ng/g Fett.
Bei den meisten Untersuchungen war das alpha-Isomer in den Proben dominierend (zwischen 60 und 95\%), so z. B. bei Carignan et al. [47], Antignac et al. [48], Colles et al. [49], Ryan et al. [50, 51] und Kakimoto et al. [45, 46]. Der erhöhte Anteil von alpha-HBCDD wird von einigen Autoren auf Unterschiede im Metabolismus zurückgeführt [52] oder auf physikochemische Eigenschaften wie z. B. eine höhere Wasserlöslichkeit [53]. Wahrscheinlicher scheint jedoch die Tatsache zu sein, dass in Produkten das alpha-Isomer dominiert und so zu einer erhöhten Exposition führt (siehe Diskussion oben).

Darüber hinaus liegen vereinzelte Nachweise von tetra- und penta-bromierten Substanzen in der Muttermilch vor [54]. Da keine radioaktiv markierten Standards vorlagen, wur- 


\begin{tabular}{|c|c|}
\hline Endpunkt & Wert \\
\hline Zustand & Fest \\
\hline Schmelzpunkt & $190^{\circ} \mathrm{C}^{\mathrm{a}}$ \\
\hline Siedepunkt & Zersetzung bei $>190^{\circ} \mathrm{C}$ \\
\hline Relative Dichte & $2,24-2,38 \mathrm{~g} / \mathrm{cm}^{3}$ \\
\hline Dampfdruck & $6,3 \times 10^{-5} \mathrm{~Pa}$ bei $21^{\circ} \mathrm{C}$ \\
\hline Verteilungskoeffizient $\log \mathrm{K}_{\mathrm{ow}}$ & $5,62^{b}$ \\
\hline Wasserlöslichkeit & $0,066 \mathrm{mg} / \mathrm{L}$ bei $20^{\circ} \mathrm{C}^{\mathrm{C}}$ \\
\hline \multicolumn{2}{|c|}{$\begin{array}{l}\text { aMittelwert, Isomere haben Werte von } 171^{\circ} \mathrm{C} \text { (beta-HBCDD), } 180^{\circ} \mathrm{C} \text { (alpha-HBCDD) und } 208^{\circ} \mathrm{C} \text { (gamma-HBCDD } \\
\text { btechnisches Produkt, Isomere haben Werte von 5,07 (alpa-HBCDD); } 5,12 \text { (beta-HBCDD) und 5,47 (gamma-HBCDD } \\
\text { cSumme der Isomere, alpha-HBCDD: 0,049 mg/L; beta-HBCDD: 0,015 mg/L und gamma-HBCDD: 0,002 mg/L. }\end{array}$} \\
\hline
\end{tabular}

\section{Tab. 2 Konzentrationen von HBCDD in der Innenraumluft}

\begin{tabular}{|llll}
\hline Land, Probenahmeort & $\begin{array}{l}\text { Anzahl der } \\
\text { Proben }\end{array}$ & $\begin{array}{l}\text { HBCDD-Gehalt: Median } \\
\left(\text { Min.-Max.) }\left[\mathrm{pg} / \mathrm{m}^{3}\right]\right.\end{array}$ & Zitat \\
\hline Schweden, Häuser & 10 & $2(<1,6-33)$ & {$[21]$} \\
\hline Schweden, Wohnungen & 44 & $-(<1,6-15)$ & {$[21]$} \\
\hline Schweden, Büros & 10 & $<1,6^{\text {a }}$ & {$[21]$} \\
\hline UK, Wohnungen & 33 & $180(67-1300)$ & {$[22]$} \\
\hline UK, Büros & 25 & $170(70-470)$ & {$[22]$} \\
\hline Japan, Häuser & 2 & $(6,7 / 10-160 / 280)$ & {$[23]$} \\
\hline
\end{tabular}

aNachweisgrenze.

\section{Tab. 3 Konzentrationen von HBCDD in Hausstaub}

\begin{tabular}{|llll}
\hline Land, Probenahmeort & $\begin{array}{l}\text { Anzahl der } \\
\text { Proben }\end{array}$ & $\begin{array}{l}\text { HBCDD-Gehalt: Median } \\
\text { (Min.-Max.) [ng/g] }\end{array}$ & Zitat \\
\hline Deutschland, Wohnungen & 20 & $345(53-4051)$ & {$[29]$} \\
\hline Schweden, Häuser & 10 & $100(15-990)$ & {$[21]$} \\
\hline Schweden, Wohnungen & 34 & $45\left(<3^{\mathrm{a}}-2400\right)$ & {$[21]$} \\
\hline Schweden, Büros & 10 & $300(190-5700)$ & {$[21]$} \\
\hline Schweden & 19 & $86\left(6,4-950.000^{\mathrm{d}}\right) / 8,9(5,9-62)$ & {$[30]$} \\
\hline Deutschland/USA, Häuser \& Wohnungen & $24 / 2$ & $166\left(30-15.000^{b}\right)$ & {$[31]$} \\
\hline Tschechien, Wohnungen & 25 & $93\left(<0,3^{\mathrm{a}}-950\right)$ & {$[24]$} \\
\hline Belgien, Häuser & 45 & $130(5-42.692)$ & {$[32]$} \\
\hline Belgien, Büros & 10 & $367(256-1153)$ & {$[31]$} \\
\hline UK, Wohnungen & 45 & $1300(140-140.000)$ & {$[22]$} \\
\hline UK, Wohnungen & 31 & $730(140-110.000)$ & {$[28]$} \\
\hline UK, Büros & 6 & $650(90-3600)$ & {$[28]$} \\
\hline UK, Wohnungen und Büros & 10 & $3250(940-6900)$ & {$[33]$} \\
\hline EU, Häuser & 12 & $-(80,3-2185)$ & {$[34]$} \\
\hline EU, Parlament, Büros & 15 & $-\left(<4^{\mathrm{a}-1400 / 3700}\right)$ & {$[35]$} \\
\hline China, Büros & 56 & $2621(652-122.973)$ & {$[36]$} \\
\hline Neuseeland, Häuser & 50 & $190(20-4100)$ & {$[37]$} \\
\hline Japan, Häuser & 2 & $-(140-13.000)$ & {$[23]$} \\
\hline Japan, Hotel & 8 & $740(72-1300)$ & {$[38]$} \\
\hline Indonesien, Häuser & 25 & $12(1,5-75)$ & {$[39]$} \\
\hline Kanada, Wohnungen & 8 & $640(64-1300)$ & {$[28]$} \\
\hline USA, Wohnungen & 37 & $230(<4,5-130.200)$ & {$[40]$} \\
\hline US, Wohnungen & 13 & $390(110-4000)$ & {$[28]$} \\
\hline US, Wohnungen & $140^{\mathrm{e}}$ & \\
\hline aNachweisgrenze. & & & \\
\hline $\begin{array}{l}\text { bnur gamma-HBCDD quantifiziert. } \\
\text { Cunterschiedliche Angaben in Abstrakt und Poster (Poster enthält mehr Daten). }\end{array}$ & \\
\hline $\begin{array}{l}\text { dhöherer Wert aus Staubsaugerbeutelproben, evtl. HBCDD-Quelle im Staubsauger. } \\
\text { earithm. Mittelwert. }\end{array}$ & & & \\
\hline
\end{tabular}

den die Konzentrationen semi-quantitativ ermittelt (Tetrabromcyclododecane (TBCDDe), Mittelwert 0,25 ng/g Fett $(n=25)$; Pentabromcyclododecane (PBCDDe), Mittelwert 0,04 ng/g Fett). Angaben zur Expositionssituation der Mütter liegen nicht vor. Da diese Stoffe aber auch als Abbauprodukte im Hausstaub nachgewiesen wurden (siehe oben), können sie entweder direkt aufgenommen worden sein oder durch Metabolismus im Menschen entstanden sein. In Ratten und Mäusen konnten diese Stoffe als Metabolite nachgewiesen werden.

\subsection{Vorkommen im Blutserum/-plasma}

In - Tab. 5 sind die Ergebnisse der Untersuchungen von Blutserum/-plasma zusammengefasst. Die meisten Werte (Median) liegen im Bereich $<0,08$ bis $10 \mathrm{ng} / \mathrm{g}$ Fett (Maximalwerte bis 52 ng/g Fett). Einzelne Proben von Arbeitern aus einem EPS herstellenden Betrieb zeigen höhere Werte (Median 101, Maximalwert 856 ng/g Fett) [72]. Auch in Korea wurden vereinzelt höhere Maximalwerte bis 166 ng/g Fett gemessen [73].

Im Rahmen einer Pilotuntersuchung von persistenten organischen Substanzen im Blut der deutschen allgemeinen Bevölkerung wurde auch HBCDD mittels LC/ MS-MS gemessen [74]. Von den 42 Proben lagen nur 7 über der Nachweisgrenze von 6 ng/g Fett, aber alle unter der Bestimmungsgrenze von 19 ng/g Fett.

Odland et al. [75] untersuchten in einer Pilotstudie das Blut von je 10 Frauen aus Bodø, Norwegen und Taimyr, Russland. Sie berichten, dass in der Hälfte der Proben HBCDD nachgewiesen wurde. Die Konzentrationen der einzelnen HBCDDIsomere liegen im Bereich von $<0,005$ bis $0,345 \mathrm{ng} / \mathrm{ml}$ Plasma. In der anschließenden Hauptstudie [76] wurden bei je 12 Frauen aus Bodø, Taimyr und Naryan Mar in nur 4 der 36 Proben Konzentrationen über der Nachweisgrenze ermittelt. Die Konzentrationen der einzelnen HBCDD-Isomere liegen im Bereich von $<0,003$ bis $<0,08 \mathrm{ng} / \mathrm{ml}$ Plasma. 
Tab. 4 Konzentrationen von HBCDD in Muttermilch (ng/g Fett)

Land, Jahr Anzahl/Anzahl > Nach-

weisgrenze

Frankreich, 2005

Belgien, 2006

Belgien, 2006

Belgien, 2009-2010

Schweden, 2008

Schweden, 1980-2004

Schweden, Uppsala, 2000-2004

Schweden, Uppsala, 1996-2006

Schweden, Göteborg, 2001

Schweden, 2003

Schweden, Lund, 2003

Schweden, Lycksele, 2003-2004

Norwegen, 1993-2001

Norwegen, 2001

Norwegen, 2000-2002

Norwegen, 2003-2005

Spanien, A Coruna, 2006-2007

Spanien, Barcelona, 2009

UK, Zeitraum nicht angegeben

USA, Texas, 2002

USA, Texas, 2002

USA, Texas, 2004

Kanada, Munavik, 1989-1991

Kanada, Munavik, 1996-2000

Kanada, Ontario, 2002-2003

Kanada, Ontario, 2003

Kanada, Ontario, 2005

Kanada, 2004-2005

Philippinen, 2004, Payatas (Müllhal-

de), Malate (Referenz)

Japan, 2007-2008

China, 2007

\section{weisgrenze}

25/7

1 (gepoolt aus 178)

22 (gepoolte Proben)

1 (gepoolt aus 84)

$18 / 17 \%$

(2)

$177 / 66 \%$

$36 / 39 \%$

5

$36 / 93 \%$

$39 / 100 \%$

$85 / 49$

$9 / 9$

$10 / 1$

$393 / 63,4 \%$

$33 / 30$

6

$34 / 34$

9

20

20

$20 / 16$

$20 / 15$

8

$27 / 13$

$35 / 23$

$43 / 43$

22,

11

$16+33,23+24$

24 (gepoolte Proben)
Alpha/beta/gamma HBCDD: Mittelwert (Min.-Max.)

$2,5-5 /-/-$

$1,5 /<0,8 /<0,8$

$3,2 / 0,05 / 0,55$

$3,2 / 0,05 / 0,55$

$<$ LOD $-122 /<$ LOD $/<$ LOD -176

$<0,2-3,6 /<1,0-3,3 /<0,3-6,2$

$3,17 / 0,30 / 0,56^{\mathrm{a}}$

$0,2-0,9 /-/-$

$0,4(0,16-0,9) /-/-$

$0,4(0,16-1,2) /-/-$

$0,2(0,1-0,6) /-/-$

$0,9(0,2-13,3) /-/-$

$0,4-19 /-1-$

$0,6(0,2-8,8) /-/-$

$0,43(0,2-28) /-/-$

$0,71(0,25-4,43) / 0,08\left(0,03-\quad 1,02^{\mathrm{b}}(0,36-8,10)\right.$

$1,64) / 0,20(0,07-3,20)$

Rural: 0,90

(LOQ-2,7)/-/-

Urban: 0,95

(LOQ-1,5)/-/-

\begin{tabular}{lllll}
\hline Hanoi, Vietnam, 2007 & $9 ;$ & $0,33(0,070-1,4)$ & $0,42(0,11-0,97)$ & {$[68]$} \\
Dong Mai; & $4 ;$ & $0,38(0,11-3,3)$ & $2,1(0,8-5,4)$ & $0,45(0,20-1,15) ;$ \\
Trang Minh & 11 & & $0,62(0,24-1,67)$ & {$[58]$} \\
\hline Mexiko, 2003 & 7 & & $10,2^{c}(<0,3-19)$ & {$[69]$} \\
\hline Russland, Murmansk, 2000; & $14 / 8 ;$ & $1,0(0,01-18), 0,62(0,03-3,2) ;$ & {$[71]$} \\
Arkhangelsk, 2002 & $23 / 3$ & $0,28(0,09-1,1) ;$ & {$[70]$} \\
\hline Australien, 1993-2009 & $13 / 9$ (gepoolte Proben) & $<0,3-10 / 3,6 /<0,3-9,2$ & $0,27(0,01-0,66)$ \\
\hline Ghana, Accra 2004, 2009; & 24,$16 ;$ & & & \\
Kumasi 2009; & $14 ;$ & & & \\
Tamale 2009 & 12 & & & \\
\hline
\end{tabular}

a Median.

${ }^{b}$ geom. Mittelwert.

carithm. Mittelwert.

dunpubl., zitiert nach Health Canada 2011.
$-(<0,32-1,5)$

Anstieg von $<0,1 \mathrm{ng} / \mathrm{g}$ Fett auf

$0,4 \mathrm{ng} / \mathrm{g}$ Fett (im Jahr 2002)

$0,3(<0,2-4,4)$

$0,25(<0,2-7,8)$

$<0,4(<0,4-2,4)$

$1,1(0,3-3,2)$

$0,4(<0,2-5,9)$

$0,4(0,09-10)$

$0,6(0,4-20)$

$-(0,25-20)$

0,13

$0,86(<0,2-31)$

27 (3-188)

$1,3-6,2$

$3,83(1,04-22,37)$

$0,89(0,15-3,2)$

$0,31(0,22-0,40)$

$1,4-1,9$

Zitat

[48]

[49]

[53]

[55]

[30]

[42]

[56]

[57]

[56]

[58]

[56]

[56]

[59]

[60]

[61]

[62]

[63]

[7]

[54]

[50]

[64] $[63,64]$ $[65]^{\mathrm{d}}$

$2,1(0,8-5,4)$

$10,2<(<0,3-19)$

$0,28(0,09-1,1)$;

$0,27(0,01-0,66)$ 


\begin{tabular}{|c|c|c|c|c|}
\hline Land, Jahr & $\begin{array}{l}\text { Anzahl/Anzahl > Nach- } \\
\text { weisgrenze }\end{array}$ & $\begin{array}{l}\text { Alpha/beta/gamma HBCDD: } \\
\text { Mittelwert (Min.-Max.) }\end{array}$ & $\begin{array}{l}\text { Gesamt HBCDD: Median } \\
\text { (Min.-Max.) }\end{array}$ & Zitat \\
\hline Deutschland, 2013 & $42 / 7$ & $<19(\mathrm{LOQ}) /<19 /<19$ & & [74] \\
\hline Kanada, Munavik NWT, 1994-1999 & 10 (gepoolt aus 560) & $0,7(0,5-0,9) /-/-$ & & {$[65]$} \\
\hline Schweden, 2000 & $50 / 44$ & & $0,46(<0,24-3,4)$ & [77] \\
\hline $\begin{array}{l}\text { Norwegen, 2004-2005, Konsumenten } \\
\text { von Fisch aus einem kontaminierten See }\end{array}$ & $\begin{array}{l}\text { Männer: } 41 ; \\
\text { Frauen: } 25\end{array}$ & & $\begin{array}{l}4,1(\mathrm{LOQ}-52) \\
2,6(\mathrm{LOQ}-18)\end{array}$ & [78] \\
\hline $\begin{array}{l}\text { Norwegen, Arbeiter in EPS Herstellung; } \\
\text { Referenzgruppe }\end{array}$ & 10 & & $\begin{array}{l}101(6-856) ; \\
<1(\text { LOD) }\end{array}$ & [72] \\
\hline Belgien, 2007 & 16 & & $<0,5-11$ & {$[25]$} \\
\hline Niederlande & $91 / 11$ & & $<0,08(<0,08-0,356)$ & [79] \\
\hline Niederlande & 78 & & $1,1(<0,2-7,0)$ & {$[25]$} \\
\hline Niederlande, 2001-2002 & $69 / 68$ & & $0,7(<0,08-7,4)$ & {$[80]$} \\
\hline Niederlande & $70^{a}+8^{b}$ & & $1,3(<0,16-7,0)$ & [81] \\
\hline Mexiko, 2003 & 5 & & $1,2(0,7-2,5)$ & [58] \\
\hline Korea, 2009-2010 & 76 & & $8,55(<M D L-165,6)$ & [73] \\
\hline
\end{tabular}

\subsection{Sonstiges Vorkommen}

Weiss et al. [81] wiesen HBCDD auch in 5 von 12 Proben Nabelschnurblut in Konzentrationen von $<0,16-4,2$ ng/g Fett (Median 0,32 ng/g Fett) nach. Ähnliche Werte berichten Meijer et al. [80] mit 0,2$4,3 \mathrm{ng} / \mathrm{g}$ Fett (Median 0,2 ng/g Fett) in 4 von 12 Proben. Demgegenüber konnten Ryan et al. [65] in 13 Proben alphaHBCDD nicht nachweisen (LOD 2,4 ng/g Fett).

Auch in menschlichem Fettgewebe wurde HBCDD in Konzentrationen bis 12 ng/g Fett nachgewiesen (Pulkrabová et al. [82]: Median <0,5 ng/g Fett, 5.95. Perzentil <0,5-7,5 ng/g Fett; Johnson-Restrepo et al. [83]: 20 Proben, Median 0,111 ng/g Fett, Min.-Max. <0,0026 -2,41 ng/g Fett; Antignac et al. [48]: 26 Proben, 1-3 ng/g Fett, 3 Proben erhöhte Werte 6-12 ng/g Fett).

In der Bewertung der EFSA [7] wird auch umfangreich auf die Ergebnisse der Analysen in Lebensmitteln eingegangen. Neuere Daten finden sich z. B. bei Goscinny et al. [84] und Törnkvist et al. [85].

\section{Allgemeines toxikologisches Profil}

\subsection{Toxikokinetik}

\subsubsection{Untersuchungen an Ratten}

Nach EU Altstoffbewertung [1,86] wird gamma-HBCDD bei Sprague-Dawley
Ratten nach einer einmaligen oralen Gabe (7-9 mg/kg KG in Olivenöl/Aceton) schnell und fast vollständig über den Gastrointestinaltrakt aufgenommen. 86\% der Dosis werden innerhalb von 3 Tagen ausgeschieden, überwiegend (70\%) über die Faeces (davon 28\% als Metabolite, $72 \%$ als nicht extrahierbare Rückstände) und teils (16\%) mit dem Urin (davon 64\% als Metabolite, $36 \%$ als nicht extrahierbare Rückstände). Die Metaboliten wurden jedoch nicht identifiziert.

Demgegenüber wird berichtet, dass Arita et al. [87] bei 4 männlichen Wistar Ratten nach einer Dosis von 500 mg/ $\mathrm{kg}$ KG/d über $5 \mathrm{~d}$ in Olivenöl, oral per Schlundsonde verabreicht (technische Mischung, feine Suspension Pyroguard SR - 103; Daiichi Kogyo Seiyaku K.K.) keine Exkretion der Ausgangssubstanz oder von Metaboliten mit dem Urin festgestellt haben (nach [1] und [2]). Die mittlere tägliche Exkretion über die Faeces lag bei 29-37\% der täglich verabreichten Dosis. Die kumulative Exkretion über die Faeces betrug 32-35\% der verabreichten Dosis. Metaboliten wurden auch in den Faeces nicht gefunden. [Hinweis: Die Studie wird auch als Ryuich et al. [88] zitiert; bei Commission on Life Sciences 2000 [89] findet sich ebenfalls dieses Zitat jedoch ohne Jahreszahl.]

Im Rahmen der 28 d Studie mit Wistar Ratten von van der Ven et al. [90] untersuchten Brandsma et al. [91] die Metaboliten im Fettgewebe, in Leber, Lunge und
Muskelgewebe der Ratten, die gegenüber technischem HBCDD in Dosen von 30 und $100 \mathrm{mg} / \mathrm{kg} \mathrm{KG/d}$ exponiert waren. Es konnten einfach hydroxylierte Metaboliten von HBCDD (OH-HBCDD) und Dihydroxy-HBCDD (diOH-HBCDD) nachgewiesen werden. Daneben wurden als debromierte Metaboliten penta- und tetra-bromierte Substanzen gefunden. Ein fünfter Metabolit, Dihydroxy-Pentabromcyclododecan, konnte nur mit GC/ MS identifiziert werden, nicht mit LC/MS (ESI -) (• Abb. 2).

Im Rahmen einer $90 \mathrm{~d}$ Studie [1]) an Ratten wurde die Verteilung der drei Stereoisomere (alpha-, beta- und gammaHBCDD) im Fettgewebe untersucht. Nach 90 Tagen wurde das alpha-Isomer in einer 12-fach höheren Konzentration als das gamma-Isomer gefunden, welches im technischen Produkt den Hauptbestandteil ausmacht. Der relative Bioakkumulationsfaktor (BAF) betrug 99 für das alphaIsomer und 11 für das beta-Isomer, wobei das gamma-Isomer den Vergleichspunkt (=1) bildete.

\subsubsection{Untersuchungen an Mäusen}

Die Unterschiede im Metabolismus der drei Stereoisomere wurden an weiblichen C57BL/6 Mäusen untersucht [92-94]. Neben verschiedenen Einzeldosen wurde auch eine wiederholte Verabreichung über mehrere Tage in die Untersuchung einbezogen sowie orale und intravenöse Gabe verglichen. Es wurde gezeigt, dass alle 3 


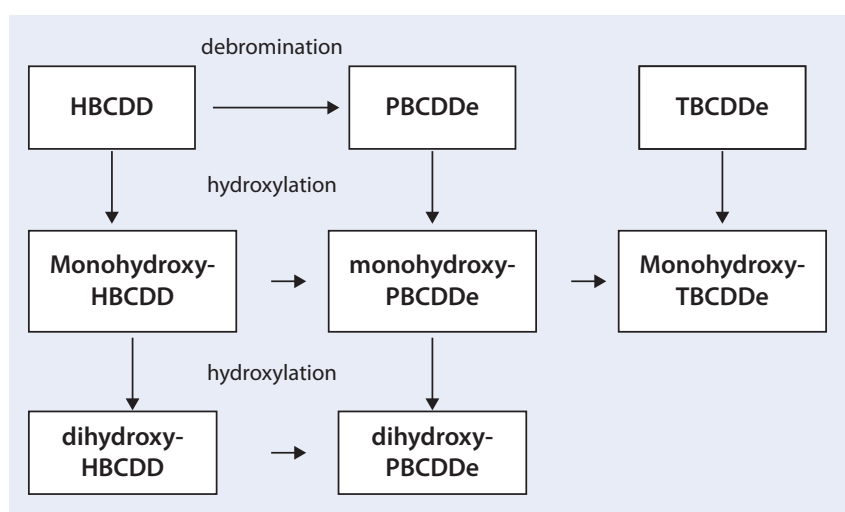

Abb. $2<$ Übersicht des Metabolismus von technischem HBCDD nach oraler Gabe bei der Ratte [91]

Isomere oral nahezu vollständig absorbiert werden. Alpha-HBCDD weist mit 17 Tagen eine wesentlich längere Halbwertszeit auf als beta- und gamma-HCBDD mit etwa 2-4 Tagen. Alpha-HBCDD reichert sich dementsprechend in höherem Umfang im Körper, insbesondere im Fettgewebe an. Die Anreicherung im Fettgewebe ist dosisabhängig (• Tab. 6).

Szabo et al. [95] untersuchten die Verteilung von alpha- und gamma-HBCDD im Gewebe von jungen sich entwickelnden Mäusen. C57BL/6 Mäuse erhielten an PND10 eine einmalige Dosis von jeweils $3 \mathrm{mg} / \mathrm{kg} \mathrm{KG}$. Es zeigte sich ein ähnlicher Verlauf wie bei adulten Mäusen, jedoch waren die Konzentrationen in den juvenilen Tieren (berechnet aus der Summe der gemessenen Organgehalte) deutlich höher im Vergleich (4 d nach der Exposition, alpha-HBCDD: juvenil $23 \%$, adult $17 \%$; gamma-HBCDD: juvenil $7 \%$, adult $<1 \%$ ).

Die Metaboliten der Untersuchungen mit alpha- und gamma-HBCDD $[92,93]$ wurden identifiziert [96]. Für alpha-HBCDD wurden einfach hydroxylierte Metaboliten von HBCDD (OHHBCDD) und nach Ringspaltung tetra- und tri-bromierte Substanzen gefunden sowie Konjugate mit Glutathion. Bei gamma-HBCDD wurden hexa- und tribromierte Carbonsäure-Reste sowie Metaboliten, die aus Debromierung und $\mathrm{Hy}$ droxylierung entstanden sind, nachgewiesen (OH-PBCDDe, diOH-PBCDDe). Hier wird eine Ringöffnung mit nachfolgender B-Oxidation vermutet. Ein Überblick ist in $\bullet$ Abb. 3 dargestellt.

\subsubsection{In-vitro-Untersuchungen}

In in-vitro-Untersuchungen [97] konnte gezeigt werden, dass technisches HBCDD sowohl Enzyme der Phase 1 als auch der Phase 2 im Metabolismus induzieren kann, insbesondere CYP2B durch Interaktion mit dem konstitutiven Androstanrezeptor (CAR) und CYP3A durch Interaktion mit dem Pregnan-X-Rezeptor (PXR).

Der Metabolismus von reinen Isomeren (- und + Enantiomere) wurde in induzierten Rattenlebermikrosomen untersucht [98]. Alpha-HBCDD und gammaHBCDD zeigten vergleichbare Halbwertszeiten (11,6-32,3 min), während für das beta-HBCDD geringere Werte (6,3 min) ermittelt wurden. Monohydroxyliertes HBCDD wurde nach der Inkubation gefunden, debromierte Metaboliten wurden nicht gefunden. Ähnliche Ergebnisse erzielten auch Huhtala et al. [99] mit induzierten Forellenlebermikrosomen.

Demgegenüber wurde in einer 1:1:1-Mischung der drei HBCDD-Isomere ebenfalls in induzierten Rattenlebermikrosomen das alpha-HBCDD nicht metabolisiert [52], während beta-HBCDD und gamma-HBCDD gut metabolisiert wurden (>60\% innerhalb von $90 \mathrm{~min}$ ). Der Metabolismus war durch CYP450 Enzyme katalysiert, da er abhängig von NADPH war.

\subsubsection{Toxikokinetik beim Menschen}

Untersuchungen zum Metabolismus beim Menschen sind nicht verfügbar.

Eine Studie zur dermalen Absorption in vitro mit menschlicher Haut (nach OECD 428 [100]) mit 9 Proben von 6 Spendern ergab, dass 31,5\% in der Hornschicht (Stratum corneum) gefunden wurden $(25,7 \%$ in den ersten 5 Klebestreifen) [101]. Aufgrund der langen lag-Phase von $4 \mathrm{~h}$ zählten die Autoren die Kle- bestreifen 11-20 zur absorbierten Dosis dazu und ermittelten so eine Absorption von $4 \%[1,101]$.

Geyer et al. [102] berechnete mit zwei unterschiedlichen Methoden eine Halbwertszeit von jeweils 64 Tagen. Bei der einen Methode wurde der Wert mit einem pharmako-kinetischen Modell aus der Körperbelastung von $700 \mathrm{ng} / \mathrm{kg}$ Fett (250-2400 ng/kg Fett) in Muttermilch und einer abgeschätzten täglichen Aufnahmemenge von 142 ng berechnet. Die Bioverfügbarkeit wurde mit $100 \%$ angenommen und die Körperfettmasse mit 13,5 kg für Männer und 18,7 kg für Frauen. Bei der zweiten Methode erfolgte die Abschätzung über die Halbwertszeit von 8 Tagen bei männlichen Ratten, dieser Wert wurde mit einem Faktor von 8 multipliziert.

\subsection{Akute Toxizität}

HBCDD zeigt eine geringe akute Toxizität nach oraler oder dermaler Verabreichung [1]. Die oralen $\mathrm{LD}_{50}$ Werte liegen bei über $20 \mathrm{~g} / \mathrm{kg}$ KG für Ratten und über 40 g/kg KG bei Mäusen. Bei Kaninchen liegt die dermale $\mathrm{LD}_{50}$ bei über $20 \mathrm{~g} / \mathrm{kg}$ KG.

In Inhalationsstudien zeigte sich bei Konzentrationen von 200 und 202 mg/L über 1-4 h keine Mortalität. Diese Untersuchungen weisen jedoch methodische Mängel (Partikelgröße nicht berichtet) auf [1].

\subsection{Haut- und Schleimhautreizung}

HBCDD zeigte eine leicht irritierende Wirkung am Auge [1]. Die Effekte waren jedoch nicht so stark, dass sie eine Einstufung als reizend zur Folge haben. Untersuchungen zur Hautreizung sowie zur Hautätzung waren negativ. Anhand der klinischen Symptome in der akuten inhalationstoxikologischen Studie gibt es keine Hinweise auf eine Reizung der Atemwege.

\subsection{Sensibilisierung}

Nach EU Altstoffbewertung [1] zeigte sich bei Untersuchungen von HBCDD aus europäischer Produktion im Magnusson-Kligman Test beim Meerschweinchen und im lokalen Lymphknoten Test 


\begin{tabular}{|c|c|c|c|}
\hline & Alpha-HBCDD & Beta-HBCDD & Gamma-HBCDD \\
\hline Einmalige Dosis & $\begin{array}{l}3,10,30,100 \mathrm{mg} / \mathrm{kg} \text { (Analyse nach } \\
4 \mathrm{~d})\end{array}$ & $\begin{array}{l}3,30,100 \mathrm{mg} / \mathrm{kg} \\
\text { (Analyse nach 4d) }\end{array}$ & $3,10,30,100 \mathrm{mg} / \mathrm{kg}$ (Analyse nach 4d) \\
\hline Mehrfache Dosis & $\begin{array}{l}10 \mathrm{~d} 3 \mathrm{mg} / \mathrm{kg} \text { (letzter Tag } 3 \mathrm{mg}{ }^{14} \mathrm{C}- \\
\text { markiert), Analyse } 4 \mathrm{~d} \text { nach letzter } \\
\text { Applikation }\end{array}$ & $\begin{array}{l}4 \mathrm{~d} 3 \mathrm{mg} / \mathrm{kg} \text { (letzter Tag } 3 \mathrm{mg}{ }^{14} \mathrm{C}- \\
\text { markiert), Analyse nach } 24 \mathrm{~h}\end{array}$ & $\begin{array}{l}10 \mathrm{~d} 3 \mathrm{mg} / \mathrm{kg} \text { (letzter Tag } 3 \mathrm{mg}{ }^{14} \mathrm{C} \text {-mar- } \\
\text { kiert), Analyse über } 14 \mathrm{~d}\end{array}$ \\
\hline Oral/iv & $\begin{array}{l}3 \mathrm{mg} / \mathrm{kg} \text { (Analyse über } 4 \mathrm{~d} \text { ), geringe } \\
\text { Unterschiede in der Elimination }\end{array}$ & $\begin{array}{l}3 \mathrm{mg} / \mathrm{kg} \text { (Analyse über } 4 \mathrm{~d} \text { ), iv weniger } \\
\text { über Urin und Faeces ausgeschieden, } \\
\text { dafür höhere Gehalte im Gewebe }\end{array}$ & $\begin{array}{l}3 \mathrm{mg} / \mathrm{kg} \text { (Analyse über } 4 \mathrm{~d} \text { ), geringe } \\
\text { Unterschiede in der Elimination }\end{array}$ \\
\hline $\begin{array}{l}\text { Verteilung der verbleibenden } \\
{ }^{14} \mathrm{C}-\text { Markierung } \\
\text { (nach } 4 \text { d) }\end{array}$ & $\begin{array}{l}\text { Hauptanteil im Fettgewebe (bis 9\%) } \\
\text { und Leber (bis 5,5\%) }\end{array}$ & $\begin{array}{l}\text { Hauptanteil im Fettgewebe (bis 6\%), } \\
\text { Muskel (bis } 2 \% \text { ) und Haut (bis 4\%) }\end{array}$ & $\begin{array}{l}\text { Nur Spuren nachweisbar: Fettgewe- } \\
\text { be }<0,01 \% \text {, Leber, Muskel und Haut } \\
0,1-0,3 \%\end{array}$ \\
\hline Halbwertszeit & $17 d$ & $2,5 d$ & $3,6 \mathrm{~d}$ \\
\hline Exkretion (über 4 Tage) & $15 \%$ Urin, $30-45 \%$ Faeces & $25-50 \%$ Urin, $50 \%$ Faeces & $25-30 \%$ Urin, $50 \%$ Faeces \\
\hline $\begin{array}{l}\text { Charakterisierung der Ex- } \\
\text { kretion }\end{array}$ & $\begin{array}{l}\text { Urin (24h): nur Metabolite } \\
\text { Faeces (24h): Ausgangssubstanz } \\
34 \% \text {, polare Metabolite } 66 \% \text { (da- } \\
\text { von } 64 \% \text { nichtextrahierbare Rück- } \\
\text { stände) }\end{array}$ & $\begin{array}{l}\text { Urin (24h): nur Metabolite } \\
\text { Faeces (24h): Ausgangssubstanz 14\%, } \\
\text { polare Metabolite } 39+30 \% \text { nichtex- } \\
\text { trahierbare Rückstände sowie } 16 \% \\
\text { gamma-Isomer }\end{array}$ & $\begin{array}{l}\text { Urin(24h): nur Metabolite } \\
\text { Faeces ( } 24 \mathrm{~h} \text { ): Ausgangssubstanz } 4 \% \text {, } \\
\text { polare Metabolite } 85 \% \text { (davon } 52 \% \\
\text { nichtextrahierbare Rückstände) sowie } \\
7 \% \text { alpha- und } 4 \% \text { beta-Isomer }\end{array}$ \\
\hline Metabolismus & $\begin{array}{l}\text { Leber (3h): Ausgangssubstanz } 62 \% \text {, } \\
38 \% \text { polare Metabolite (davon } 38 \% \\
\text { nichtextrahierbare Rückstände) } \\
\text { Fettgewebe (24h): } 100 \% \text { Ausgangs- } \\
\text { substanz, keine Isomerisation }\end{array}$ & $\begin{array}{l}\text { Leber (3h): Ausgangssubstanz 0,3\%, } \\
\text { polare Metabolite } 66 \text { und } 33 \% \text { nicht- } \\
\text { extrahierbare Rückstände } \\
\text { Fettgewebe (8h): Ausgangssubstanz } \\
92 \% \text {, andere Isomere in geringen } \\
\text { Mengen (je 3\%) }\end{array}$ & $\begin{array}{l}\text { Leber }(3 \mathrm{~h}) \text { : Ausgangssubstanz } 6 \% \text {, pola- } \\
\text { re Metabolite } 79 \% \text { (davon } 17 \% \text { nichtex- } \\
\text { trahierbare Rückstände) } \\
\text { Fettgewebe }(24 \mathrm{~h}) \text { : } \\
\text { Ausgangssubstanz } 84 \% \text { andere Isomere } \\
(5 \text { und } 11 \%)\end{array}$ \\
\hline
\end{tabular}

(local lymph node assay = LLNA) bei der Maus kein Hinweis auf eine sensibilisierende Wirkung. Demgegenüber ist HBCDD aus japanischer Produktion im Magnusson-Kligman Test positiv getestet worden.

In der australischen Bewertung [2] wird eine Human-Studie mit je 10 weiblichen und männlichen Freiwilligen berichtet. Hier führte die Applikation von $10 \%$ igem HBCDD auf der Haut zu keiner Reaktion. Die Beobachtungszeit betrug 3 Wochen. Weitere Hinweise sind nicht verfügbar.

In Bezug auf eine Atemwegs-sensibilisierende Wirkung liegen keine Hinweise vor.

\subsection{Toxizität bei wiederholter Verabreichung}

Die Wirkung von HBCDD nach wiederholter oraler Verabreichung wurde bei Ratten und Mäusen in subakuten, subchronischen und chronischen Studien untersucht (• Tab. 7).

Die Studienergebnisse werden in den Bewertungen verschiedener Gremien ausführlich dargestellt und diskutiert [1, 2]. Im Gegensatz zu den Schlussfolgerungen der Autoren der Studienberich- te werden im Rahmen der Bewertungen z. T. deutlich niedrigere NOAEL-Werte abgeleitet, da die Effekte auf das Lebergewicht zusammen mit anderen Effekten auf die Schilddrüse als advers angesehen werden. Die EU [1] schlägt die $\mathrm{BMDL}_{20}$ von $22,9 \mathrm{mg} / \mathrm{kg} \mathrm{KG} / \mathrm{d}$ für Effekte auf das Lebergewicht aus der 28d-Studie von van der Ven et al. [90] als Ausgangspunkt für die Risikobewertung vor. NICNAS [2] leiten aus dieser Studie einen NOAEL von $10 \mathrm{mg} / \mathrm{kg} \mathrm{KG/d} \mathrm{ab,} \mathrm{basierend} \mathrm{auf} \mathrm{den} \mathrm{er-}$ höhten Lebergewichten bei Weibchen ab einer Dosis von $30 \mathrm{mg} / \mathrm{kg} \mathrm{KG/d}$.

\subsection{Gentoxizität}

Zur genotoxischen Wirkung von HBCDD liegen in-vitro- als auch in-vivo-Studien vor.

\subsubsection{In vitro}

In verschiedenen Ames-Tests mit Salmonella typhimurium war HBCDD nicht mutagen [1, 108-114]. Es wurden die Stämme TA1535, TA1537, TA100, TA1538 und TA98, TA 100 jeweils mit und ohne metabolische Aktivierung (Arochlor-induzierter Rattenleber S9Mix) untersucht. Die Substanz wurde in
DMSO gelöst und in Konzentrationen bis $300 \mu \mathrm{g} /$ Platte getestet.

In einem HPRT-Genmutationstest, der nicht nach den Vorgaben der entsprechenden Richtlinie durchgeführt wurde, wurden 2 Zelllinien des Chinesischen Hamsters (Sp5/V79 und SDP8) untersucht. Die Substanz wurde in DMSO gelöst und in Konzentrationen von 3 bis $20 \mu \mathrm{g} / \mathrm{mL}$ getestet. Es wurde eine geringe aber statistisch signifikante Erhöhung der somatischen Rekombinationen (Sp5/ V79: 2,2fach und SDP8: 1,9fach) beobachtet $[1,115]$. Diese Erhöhung ist im Vergleich zu anderen klar positiv wirkenden Stoffen z. B. Cr(VI) gering und die biologische Relevanz für die in-vivo-Situation ist unklar [1, 2].

An humanen Lymphozyten aus dem peripheren Blut wurden Untersuchungen auf chromosomenschädigende Effekte durchgeführt (nach OECD Richtlinie 473) [116]. Es wurden mit und ohne metabolische Aktivierung bei Konzentrationen bis $2500 \mu \mathrm{g} / \mathrm{mL}$ keine Effekte beobachtet $[1,117])$.

\subsubsection{In vivo}

HBCDD induzierte keine Mikrokerne in der Maus [1, 118]). Mäusen (CD-1, je 4 Weibchen und Männchen) wurden Do- 


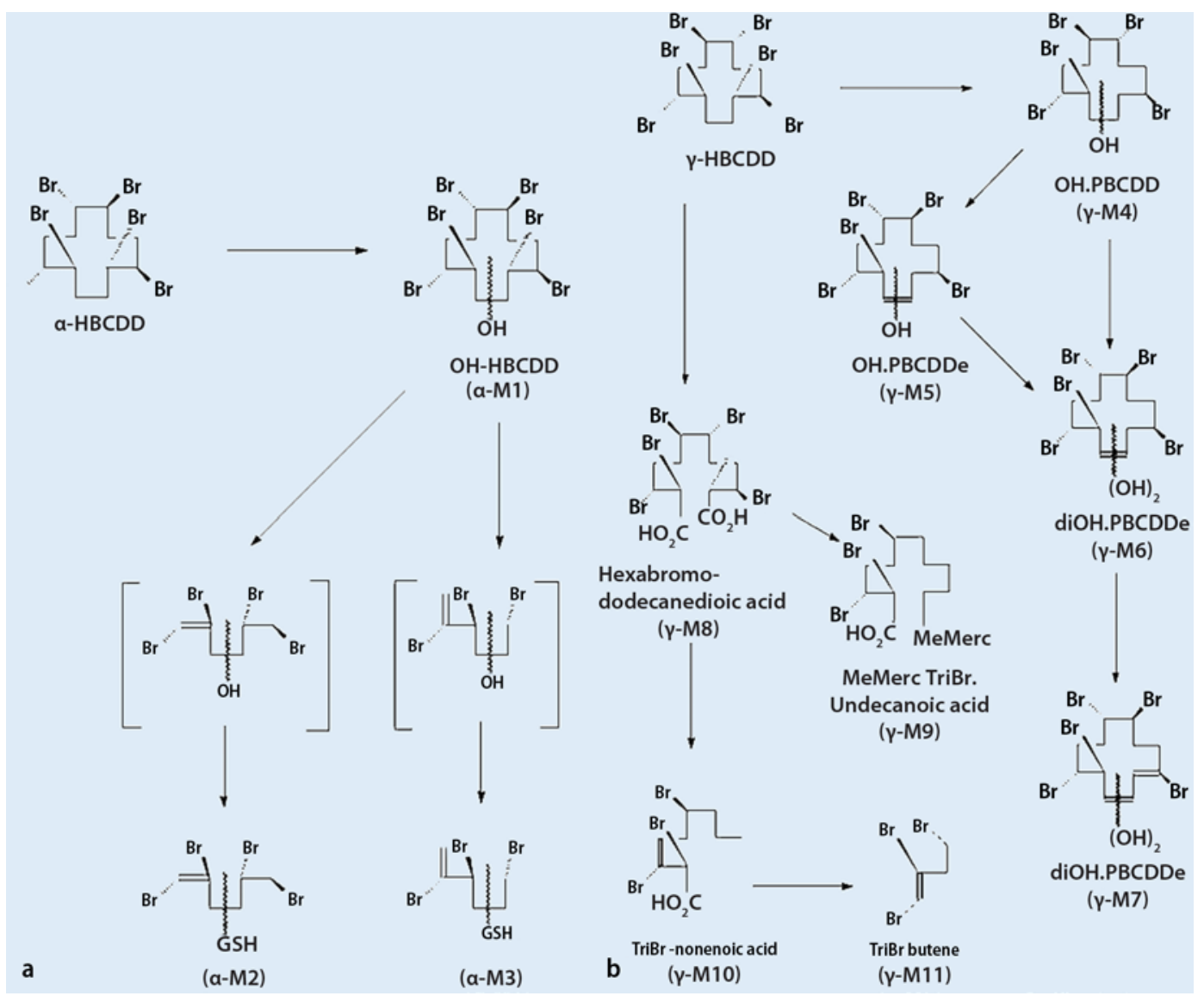

Abb. $3<$ Übersicht des Metabolismus von alpha-HBCDD und gamma-HBCDD nach einmaliger oraler Gabe bei der Maus [96] sen von 500, 1000 und $2000 \mathrm{mg} / \mathrm{kg} \mathrm{KG}$ zweimal im Abstand von 24 h verabreicht. Nach weiteren 24 h wurde das Knochenmark präpariert. Die Rate an Mikrokernen war nicht erhöht.

\subsection{Kanzerogenität}

Nach EU Altstoffprogramm [1] wurden von Kurokawa et al. [119] B6C3F1 Mäuse (je 50 pro Geschlecht und Dosisgruppe) über 18 Monate mit HBCDD in Konzentrationen von $0,100,1000$ und $10.000 \mathrm{ppm}$ (entsprechend 0, 13, 130 und $1300 \mathrm{mg} /$ $\mathrm{kg} \mathrm{KG/d)} \mathrm{gefüttert.} \mathrm{Es} \mathrm{lag} \mathrm{für} \mathrm{die} \mathrm{Bewer-}$ tung durch die EU nur eine Studienzusammenfassung vor, so dass wesentliche Validitätskriterien nicht geprüft werden konnten. Die Mortalität war im Vergleich zur Kontrolle nicht statistisch signifikant erhöht. Das Körpergewicht war in beiden Geschlechtern nicht beeinflusst. Als Haupteffekt wurden histopathologische Leberveränderungen beobachtet (• Tab. 8). Hepatozytenschwellung, Degeneration, Nekrose, Vakuolenbildung und Fettinfiltration können auf eine In- duktion von Leberenzymen hinweisen. Es bestand eine geringe Korrelation zwischen Dosis und Effekt.

\subsection{Wirkung auf die Reproduktion und die Entwicklung}

Die Studien zur Wirkung von HBCDD auf die Reproduktion und die embryonale Entwicklung sind in den folgenden Kapiteln zusammenfassend dargestellt (siehe auch • Tab. 10). Ausführliche Beschreibungen finden sich in der EU Altstoffbewertung [1] sowie in den Begründungen der ECHA [5, 6]. Zusätzliche Informationen zu den Studien sind bei NICNAS [2] und ACC [120] verfügbar.

\subsubsection{Reproduktionstoxizität}

In einer 2-Generationenstudie an Ratten wurden Effekte auf die Reproduktion untersucht [121]. Ratten (Sprague Dawley Crl:CD, 24 Tiere pro Geschlecht und Dosisgruppe) wurden Konzentrationen von 150, 1500 und 15.000 ppm im Futter über einen Zeitraum von 10 Wochen vor der Verpaarung der F0 Generation bis zum
Verpaaren der F1 Generation (Tag 88 nach der Geburt) verabreicht. Aus dem Futterverbrauch wurde eine interne Dosis von $10,2 / 14,0 ; 101 / 141$ und 1008/1363 mg/kg KG/d für Männchen/Weibchen der F0Generation undvon 11,4/14,3;115/138 und 1142/1363 mg/kg KG/d für Männchen/ Weibchen der F1-Generation abgeschätzt. Mortalität wurde bei den F0 und F1 Elterntieren nicht beobachtet. Klinische Parameter zeigten keinen Effekt. Das Körpergewicht war bei den F1 Elterntieren in der hohen Dosisgruppe reduziert, ebenso die Futteraufnahme. Der Fertilitätsindex war nicht beeinflusst, obwohl es einige als marginal eingestufte Effekte (um $10 \%$ ) in der F0 Generation bei der hohen Dosisgruppe gab (Anteil trächtige Weibchen im Vergleich zur Gesamtanzahl verpaarter Weibchen, Anteil Muttertiere mit lebenden Nachkommen im Vergleich zur Gesamtanzahl verpaarter Weibchen), die jedoch in der F1 bei gleicher Dosis über einen längeren Zeitraum nicht beobachtet wurden. Dieser Effekt wurde daher als nicht relevant eingestuft und auf mögliche Sekundäreffekte durch die beobach- 


\begin{tabular}{|c|c|c|c|c|c|}
\hline $\begin{array}{l}\text { Spezies, Stamm, Ge- } \\
\text { schlecht }^{\mathrm{a}} \text {, Anzahl }{ }^{\mathrm{b}}\end{array}$ & Dauer [d] & Dosierung & NOAELC $[\mathrm{mg} / \mathrm{kg} \mathrm{KG/d]}$ & Effekte/Anmerkungen & Zitat \\
\hline $\begin{array}{l}\text { Ratte, Sprague } \\
\text { Dawley, } \\
\mathrm{m} / \mathrm{w}, 10 / 10\end{array}$ & 28 & $\begin{array}{l}0,10.000,25.000 \\
50.000 \mathrm{ppm} \text { im Futter }(0,940, \\
2410,4820 \mathrm{mg} / \mathrm{kg} \mathrm{KG} / \mathrm{d})\end{array}$ & LOAEL 940 & Lebergewicht $\uparrow$ & {$[1,103]$} \\
\hline $\begin{array}{l}\text { Ratte, Sprague-Daw- } \\
\text { ley, } \mathrm{m} / \mathrm{w}, 6 ; 12 / 6 ; 12\end{array}$ & $\begin{array}{l}28+14 \text { Erho- } \\
\text { lung }\end{array}$ & $\begin{array}{l}125,350,1000 \mathrm{mg} / \mathrm{kg} \mathrm{KG} / \mathrm{d} \\
\text { oral per Schlundsonde in } \\
\text { Maiskeimöl }\end{array}$ & 350 & $\begin{array}{l}\text { Lebergewicht } \uparrow \text { w, nach Erholungs- } \\
\text { phase }\end{array}$ & {$[1,104]$} \\
\hline Ratte, Wistar, m/w, $5 / 5$ & 28 & $\begin{array}{l}0 ; 0,3 ; 1 ; 3 ; 10 ; 30 ; 100 ; \\
200 \text { mg/kg KG/d oral per } \\
\text { Schlundsonde in Maiskeimöl }\end{array}$ & $\begin{array}{l}\text { Lebergewicht } \mathrm{BMDL}_{20} \text { : } \\
22,9 \text { mg/kg KG/d } \\
\text { Schilddrüsengewicht } \\
\mathrm{BMDL}_{10}: 1,6 \mathrm{mg} / \mathrm{kg} \\
\mathrm{KG} / \mathrm{d}\end{array}$ & $\begin{array}{l}\text { Auswertung per Benchmark-Ver- } \\
\text { fahren, Schilddrüsengewicht } \downarrow \text {, } \\
\text { T4 } \downarrow \text {, } \\
\text { T4 UGT } \uparrow \text {, Hypophysengewicht } \uparrow \text {, } \\
\text { Splenozyten (Monozyten in der } \\
\text { Milz) Anzahl } \downarrow \text {, } \\
\text { Lebergewicht } \uparrow\end{array}$ & [90] \\
\hline Ratte, Wistar, m/w, 5/5 & 28 & $\begin{array}{l}0 ; 0,3 ; 1 ; 3 ; 10 ; 30 ; 100 ; \\
200 \text { mg/kg KG/d oral per } \\
\text { Schlundsonde in Maiskeimöl }\end{array}$ & $\begin{array}{l}\text { CYP3A } \\
1(\mathrm{w}), 10(\mathrm{~m}) \\
\text { CYP2B } \\
10(\mathrm{w}), 30(\mathrm{~m})\end{array}$ & $\begin{array}{l}\text { Induktion von Leberenzymen, ver- } \\
\text { mehrt in Weibchen }\end{array}$ & [97] \\
\hline Maus, BALB/c, w, 6-7 & 28 & $\begin{array}{l}\text { 0, } 10.000 \mathrm{ppm} \text { im Futter } \\
(1700 \mathrm{mg} / \mathrm{kg} \mathrm{KG} / \mathrm{d})\end{array}$ & 1700 & $\begin{array}{l}\text { Keine Erhöhung des pulmonalen } \\
\text { Virus-Titers nach Infektion mit } \\
\text { Respiratorischen-Synzytial-Viren (RSV) }\end{array}$ & [105] \\
\hline $\begin{array}{l}\text { Ratte, Sprague-Daw- } \\
\text { ley, } \mathrm{m} / \mathrm{w}, 20 / 20\end{array}$ & $\begin{array}{l}90+42 \text { Erho- } \\
\text { lung }\end{array}$ & $\begin{array}{l}0,1600,3200,6400, \\
128.000 \mathrm{ppm} \text { im Futter (0, } \\
120,240,470,950 \mathrm{mg} / \mathrm{kg} \\
\mathrm{KG} / \mathrm{d})\end{array}$ & $\begin{array}{l}950^{c} \\
\text { LOAEL } 120\end{array}$ & $\begin{array}{l}\text { Rel. Lebergewichte } \uparrow \text { (in allen Do- } \\
\text { sisgruppen, w: 5-27\%, m: 8-22\%) }\end{array}$ & {$[1,106]$} \\
\hline $\begin{array}{l}\text { Ratte, Sprague-Daw- } \\
\text { ley, } \mathrm{m} / \mathrm{w}, 15 / 15\end{array}$ & $\begin{array}{l}90+28 \text { Erho- } \\
\text { lung }\end{array}$ & $\begin{array}{l}0,100,300,1000 \mathrm{mg} / \mathrm{kg} \mathrm{KG} / \mathrm{d} \\
\text { oral per Schlundsonde in } \\
\text { Maiskeimöl }\end{array}$ & $\begin{array}{l}1000^{c} \\
\text { LOAEL } 100\end{array}$ & $\begin{array}{l}\text { Rel. Lebergewichte } \uparrow \text { (in allen } \\
\text { Dosisgruppen, w: } 24-48 \%, \mathrm{m:} \\
\text { 18-44\%), Serum T4 } \downarrow \text { m, TSH } \uparrow \text { w }\end{array}$ & {$[1,107]$} \\
\hline \multicolumn{6}{|c|}{$\begin{array}{l}\text { T4 Thyroxin; TSH Thyroidea stimulierendes Hormon, UGT UDP-Glucuronosyltransferasen. } \\
\text { am: Männchen, w:Weibchen. } \\
\text { bAnzahl Männchen/Weibchen je Dosisgruppe. } \\
\text { cAbleitung des NOAEL durch die Autoren der Studie. }\end{array}$} \\
\hline
\end{tabular}

Tab. 8 Ergebnisse der histopathologischen Untersuchung der Leber im Rahmen der chroni-

schen Fütterungsstudie an Mäusen [1, 119]

\begin{tabular}{|c|c|c|c|c|}
\hline Befund & $\begin{array}{l}\text { Kont- } \\
\text { rolle }\end{array}$ & $\begin{array}{l}13 \mathrm{mg} / \mathrm{kg} \\
\mathrm{KG} / \mathrm{d}\end{array}$ & $\begin{array}{l}130 \mathrm{mg} / \mathrm{kg} \\
\mathrm{KG} / \mathrm{d}\end{array}$ & $1300 \mathrm{mg} / \mathrm{kg} \mathrm{KG} / \mathrm{d}$ \\
\hline Leberzellkarzinom m/w & $12 / 0$ & $17 / 0$ & $25 / 1$ & $13 / 5$ \\
\hline Hämangiom m/w & $2 / 1$ & $2 / 0$ & $2 / 0$ & $0 / 1$ \\
\hline Veränderte Foci m/w & $20 / 12$ & $20 / 6$ & $41 / 10$ & $25 / 10$ \\
\hline $\begin{array}{l}\text { Hepatozytenschwel- } \\
\text { lung } \mathrm{m} / \mathrm{w}\end{array}$ & $29 / 37$ & & & \\
\hline
\end{tabular}

tete Schilddrüsen- und Lebertoxizität zurückgeführt [6]. Alle anderen Parameter zeigten keinen Effekt auf die Reproduktion oder Entwicklung der Nachkommen. Weitere Effekte auf die Elterntiere in der F0 und F1 Generation werden in der - Tab. 9 dargestellt.

Die Effekte auf die Primordialfollikel sind nicht eindeutig, da sie sich noch im Bereich der historischen Kontrollen bewegen und dieser Endpunkt bei Ratten eine hohe Variabilität aufweist [5]. Es wurde ein NOAEL von 10,2 mg/kg KG/d (150 ppm) festgelegt.

In einer 1-Generationen Reproduktionsstudie [122] mit einer erhöhten Anzahl an Dosisgruppen und verringerter Tieranzahl pro Gruppe wurden die Effekte mit einem Benchmarkdosis-Ansatz ausgewertet. Ratten Wochen (Wistar, 10 Tiere pro Geschlecht und Dosisgruppe) wurden Konzentrationen von 0; 0,1; 0,3; 1; 3; 10; 30 und $100 \mathrm{mg} / \mathrm{kg} \mathrm{KG/d} \mathrm{im}$ Futter über einen Zeitraum von 10 Wochen (Männchen) bzw. 2 Wochen (Weib- chen) vor der Verpaarung der F0 Generation bis zum Entwöhnen der F1 Generation (Postnatalwoche 3 - PNW3) verabreicht. Die F1 Tiere wurden insgesamt 11 Wochen (PNW11) exponiert und anschließend untersucht. Die Gewichtsreduktion der Testes war der empfindlichste Parameter in Bezug auf die Reproduktion mit einer $\mathrm{BMDL}_{5}$ von $1,5 \mathrm{mg} /$ $\mathrm{kg} \mathrm{KG/d} \mathrm{(interne} \mathrm{Dosis} 52 \mu \mathrm{g} / \mathrm{g}$ Leberfett). Das Körpergewicht war reduziert in der F1 Generation (BMDL 10 : 62,7 mg/ $\mathrm{kg} \mathrm{KG/d}$ für Männchen bzw. 57,9 mg/kg KG/d für Weibchen). Bei Männchen der F1 Generation war die IgG Antwort erhöht mit einer $\mathrm{BMDL}_{20}$ von $0,46 \mathrm{mg} / \mathrm{kg}$ KG/d. Dieser Effekt zeigte keine DosisWirkungsbeziehung. Aufgrund der hohen Variabilität des Effektes ist die gewählte kritische Effektgröße von $20 \%$ bedeutungslos; solche BMDL-Werte sollten daher mit Vorsicht betrachtet werden [5]. Bei Weibchen der F1 Generation war der empfindlichste Endpunkt die Knochen- 


\begin{tabular}{|c|c|c|c|c|}
\hline Dosis [ppm] & Weibliche F0 Tiere & Männliche F0 Tiere & Weibliche F1 Tiere & Männliche F1 Tiere \\
\hline \multirow[t]{6}{*}{15.000} & $\begin{array}{l}\text { Inzidenz verringerte Schild- } \\
\text { drüsenfollikelgröße } \uparrow\end{array}$ & $\begin{array}{l}\text { Inzidenz verringerte Schild- } \\
\text { drüsenfollikelgröße } \uparrow\end{array}$ & $\begin{array}{l}\text { Inzidenz verringerte Schilddrüsen- } \\
\text { follikelgröße } \uparrow\end{array}$ & $\begin{array}{l}\text { Inzidenz verringerte Schilddrü- } \\
\text { senfollikelgröße } \uparrow\end{array}$ \\
\hline & Serum TSH $\uparrow$ & - & Serum TSH $\uparrow$ & \\
\hline & Serum T4 $\downarrow$ & Serum T4 $\downarrow$ & & \\
\hline & & & Anzahl Primordialfollikel $\downarrow$ & \\
\hline & & & $\begin{array}{l}\text { Lebergewicht (abs./rel) } \uparrow \text { adulte als } \\
\text { auch frisch entwöhnte Tiere (wean- } \\
\text { lings) }\end{array}$ & $\begin{array}{l}\text { Lebergewicht (abs./rel) } \uparrow \text { adulte } \\
\text { als auch frisch entwöhnte Tiere } \\
\text { (weanlings) }\end{array}$ \\
\hline & & & Körpergewicht und Futterverbrauch $\downarrow$ & $\begin{array}{l}\text { Körpergewicht und Futterver- } \\
\text { brauch } \downarrow\end{array}$ \\
\hline \multirow[t]{4}{*}{1500} & $\begin{array}{l}\text { Inzidenz verringerte Schild- } \\
\text { drüsenfollikelgröße } \uparrow\end{array}$ & $\begin{array}{l}\text { Inzidenz verringerte Schild- } \\
\text { drüsenfollikelgröße } \uparrow\end{array}$ & $\begin{array}{l}\text { Inzidenz verringerte Schilddrüsen- } \\
\text { follikelgröße } \uparrow\end{array}$ & $\begin{array}{l}\text { Inzidenz verringerte Schilddrü- } \\
\text { senfollikelgröße } \uparrow\end{array}$ \\
\hline & Serum TSH $\uparrow$ & & Serum TSH $\uparrow$ & \\
\hline & & & Anzahl Primordialfollikel $\downarrow$ & \\
\hline & & $\begin{array}{l}\text { Lebergewicht (abs./rel) } \uparrow \\
\text { adulte als auch frisch ent- } \\
\text { wöhnte Tiere (weanlings) }\end{array}$ & $\begin{array}{l}\text { Lebergewicht (abs./rel) } \uparrow \text { nur frisch } \\
\text { entwöhnte Tiere (weanlings) }\end{array}$ & $\begin{array}{l}\text { Lebergewicht (abs./rel) } \uparrow \text { nur } \\
\text { frisch entwöhnte Tiere (wean- } \\
\text { lings) }\end{array}$ \\
\hline 150 & Serum TSH $\uparrow$ & Keine Effekte & Keine Effekte & Keine Effekte \\
\hline
\end{tabular}

Tab. 10 Übersicht der Studien zur Wirkung von HBCDD auf Reproduktion und Entwicklung

\begin{tabular}{|c|c|c|c|c|}
\hline Art der Studie & Dosisgruppen & Effekte, Anmerkungen & NOAEL & Zitat \\
\hline 2-Generationen-Studie, Ratte & $\begin{array}{l}\text { 150, } 1500 \text { und } 15.000 \text { ppm } \\
\text { im Futter }\end{array}$ & $\begin{array}{l}\text { Lebergewicht } \uparrow \text {, Fertilitätsindex reduziert } \\
\text { in F0 (nicht sign.), Anzahl Primordialfollikel } \\
\text { in F1w } \downarrow\end{array}$ & $150 \mathrm{ppm}(10,2 \mathrm{mg} / \mathrm{kg} \mathrm{KG} / \mathrm{d})$ & [121] \\
\hline 1-Generationen-Studie, Ratte & $\begin{array}{l}0 ; 0,1 ; 0,3 ; 1 ; 3 ; 10 ; 30 ; \\
100 \mathrm{mg} / \mathrm{kg} \mathrm{KG} / \mathrm{d}\end{array}$ & $\begin{array}{l}\text { m: Gewichtsreduktion der Testes, } \mathrm{BMDL}_{5}: \\
\text { 1,5 mg/kg KG/d; w: Knochendichte } \mathrm{BMDL}_{10} \text { : } \\
\text { 0,056 mg/kg KG/d; } \\
\text { m + w: Körpergewicht, } \mathrm{BMDL}_{10}: 60 \text { mg/kg } \\
\mathrm{KG} / \mathrm{d}\end{array}$ & $\begin{array}{l}\text { Nicht abgeleitet, BMD- } \\
\text { Verfahren angewendet }\end{array}$ & {$[122]$} \\
\hline Entwicklungstoxizität, Ratte & $\begin{array}{l}0,100,1000,10.000 \mathrm{ppm}(0, \\
5,50,500 \mathrm{mg} / \mathrm{kg} \mathrm{KG} / \mathrm{d})\end{array}$ & $\begin{array}{l}\text { Keine Effekte auf die Entwicklung, erhöhtes } \\
\text { Lebergewicht bei } 10.000 \text { ppm }\end{array}$ & 1000 ppm (50 mg/kg KG/d) & [124] \\
\hline Entwicklungstoxizität, Ratte & $\begin{array}{l}0,250,500,1000 \mathrm{mg} / \mathrm{kg} \\
\mathrm{KG} / \mathrm{d}\end{array}$ & $\begin{array}{l}\text { Keine Effekte auf die Entwicklung, keine } \\
\text { sonstigen Effekte }\end{array}$ & $1000 \mathrm{mg} / \mathrm{kg} \mathrm{KG} / \mathrm{d}$ & [125] \\
\hline Entwicklungstoxizität, Ratte & $\begin{array}{l}0,100,1000,10.000 \mathrm{ppm} \\
{[8-21,81-213 \text { und } 803-} \\
2231 \mathrm{mg} / \mathrm{kg} \mathrm{KG} / \mathrm{d}]\end{array}$ & $\begin{array}{l}\text { Keine Effekte auf die Entwicklung, erhöhtes } \\
\text { Lebergewicht, erhöhtes Gewicht der Schild- } \\
\text { drüse }\end{array}$ & $\begin{array}{l}100 \mathrm{ppm}(8-21 \mathrm{mg} / \mathrm{kg} \\
\mathrm{KG} / \mathrm{d})\end{array}$ & [126] \\
\hline Entwicklungstoxizität, Ratte & $0,100,1000,10.000 \mathrm{ppm}$ & Keine Effekte auf die Entwicklung & & [127] \\
\hline $\begin{array}{l}\text { Entwicklungsneurotoxizität, } \\
\text { Maus }\end{array}$ & $\begin{array}{l}0,9 \text { und } 13,5 \mathrm{mg} / \mathrm{kg} \mathrm{KG} \text { als } \\
\text { einmalige Dosis an PND } 10\end{array}$ & $\begin{array}{l}\text { Effekte auf Spontanbewegung sowie Lern- } \\
\text { und Gedächtnisfähigkeit }\end{array}$ & $0,9 \mathrm{mg} / \mathrm{kg} \mathrm{KG}(=\mathrm{LOAEL})$ & [128] \\
\hline
\end{tabular}

mineraldichte der Schienbeintrabekel mit einer $\mathrm{BMDL}_{10}$ von $0,056 \mathrm{mg} / \mathrm{kg} \mathrm{KG/d}$. Die maximale Reduktion betrug 22,6\%. Die niedrige BMDL kann an der Tatsache liegen, dass die Maiskeimölkontrolle eine signifikant höhere Knochenmineraldichte aufweist $\left(294 \pm 19 \mathrm{mg} / \mathrm{cm}^{2}\right)$ als die Standardkontrolle $\left(245 \pm 27 \mathrm{mg} / \mathrm{cm}^{2}\right)$. Alle andere Effekte in Bezug auf Knochen zeigten erst Effekte bei höheren Dosen $\left(\mathrm{BMDL}_{10}>50 \mathrm{mg} / \mathrm{kg} \mathrm{KG/d}\right)$. Effekte auf die Schilddrüse wurden in dieser Studie nicht beobachtet.

Aus der oben genannten Studie wurden je 5 Tiere pro Geschlecht und Grup- pe zum Zeitpunkt Tag 110 in einem Test auf Katalepsie (Verharren in starrer Körperhaltung) und zum Zeitpunkt Tag 140 in einem Test auf akustisch evozierte Hirnstammpotentiale (BAEP) untersucht [123]. Für den Endpunkt Katalepsie wurden $\mathrm{BMDL}_{20}$-Werte von 0,6-4,4 mg/kg KG/d für Männchen und Weibchen ermittelt, für den Endpunkt akustisch evozierte Hirnstammpotentiale $\mathrm{BMDL}_{20^{-}}$ Werte von $0,2-46,7 \mathrm{mg} / \mathrm{kg} \mathrm{KG} / \mathrm{d}$ nur für Männchen. Weibchen zeigten keine signifikanten Unterschiede.

\subsubsection{Entwicklungstoxizität}

Bewertungsrelevante Studien zur Wirkung von HBCDD auf die embryonale Entwicklung wurden an Ratten durchgeführt.

Trächtigen Ratten (Crl: CD(SD) IGS Br, $n=25$ ) wurden während Tag 6 bis $19 \mathrm{der}$ Trächtigkeit täglich Dosen von 0, 250, 500 und $1,000 \mathrm{mg} / \mathrm{kg} \mathrm{KG/d}$ (in Maiskeimöl) oral per Schlundsonde verabreicht $[2,120$, 125]. Die Studie wurde nach OECD Richtlinie 414 [129] durchgeführt. Das Körpergewicht war unbeeinflusst und es wurde keine Mortalität beobachtet. Klinische Parameter zeigten keinen Effekt. Es wur- 


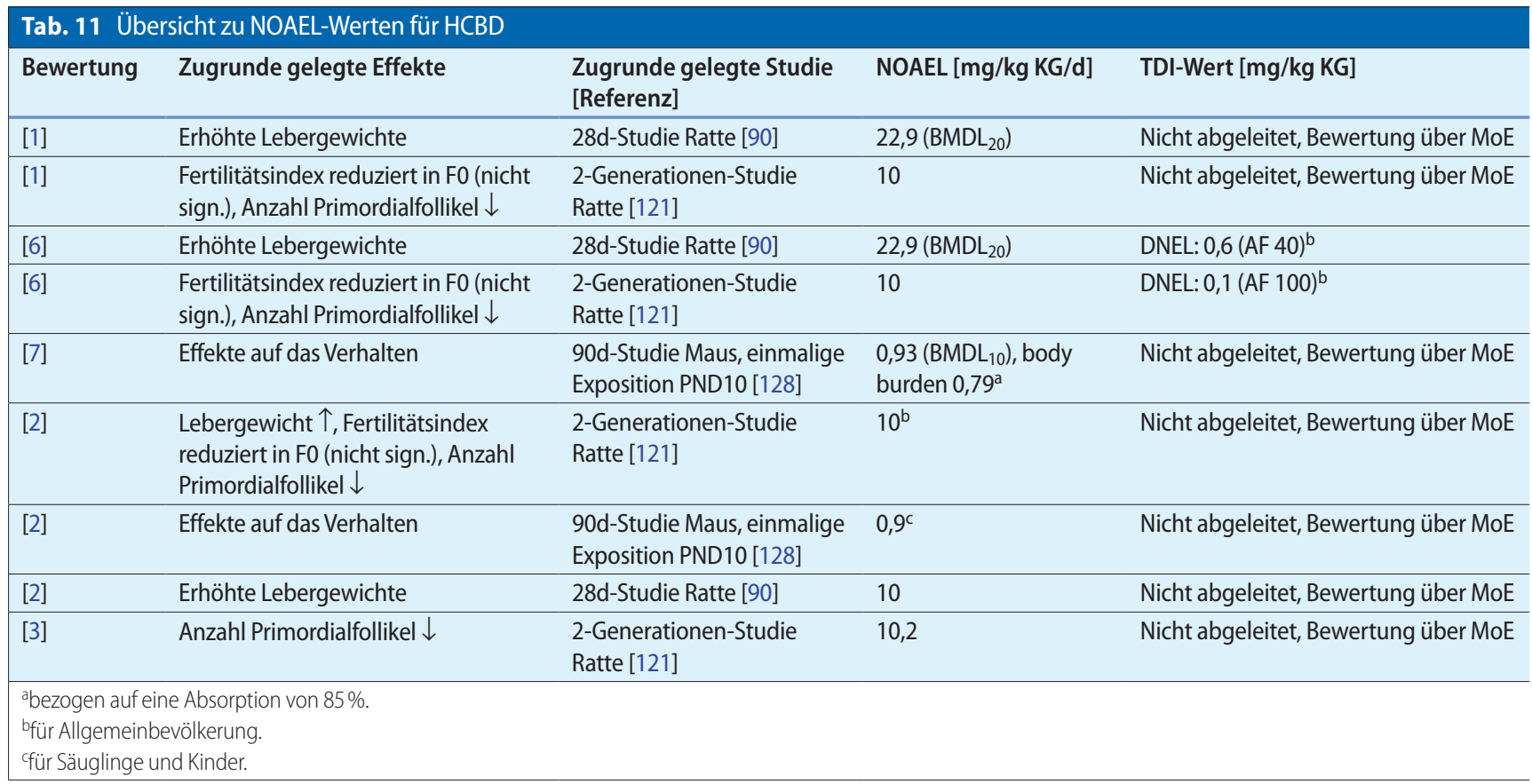

den keine maternaltoxischen und entwicklungstoxischen Effekte beobachtet. Der NOAEL für beide Endpunkte liegt bei $1000 \mathrm{mg} / \mathrm{kg} \mathrm{KG/d}$.

In einer weiteren Studie wurde trächtigen Ratten (Wistar, $n=20$ ) während Tag 6 bis 20 der Trächtigkeit täglich Futter mit Konzentrationen von 0, 100, 1000 und 10.000 ppm HBCDD (angegeben als 0; 0,01; 0,1 und $1 \%$ ) verabreicht. Den Autoren zufolge entspricht dies 0, 5, 50 und $500 \mathrm{mg} /$ $\mathrm{kg} \mathrm{KG/d}$, allerdings finden sich in einigen Übersichtsarbeiten hierzu Schätzwerte von $0 ; 7,5 ; 75$ und $750 \mathrm{mg} / \mathrm{kg} \mathrm{KG/d} \mathrm{ba-}$ sierend auf einem mittleren Rattengewicht von $200 \mathrm{~g}$ und einem mittleren Futterverbrauch von $15 \mathrm{~g} / \mathrm{d}$ [2, 120, 124]. 14 Tiere wurden an Tag 20 getötet und 6 Tiere gebaren die Nachkommen natürlich, welche bis zum Entwöhnen über 6 Wochen weiter beobachtet wurden. Es wurde keine Mortalität beobachtet. In der höchsten Dosisgruppe war der Futterverbrauch reduziert und bei den getöteten Tieren wurde ein um $13 \%$ erhöhtes Lebergewicht (abs./rel.) ermittelt. Es wurden keine entwicklungstoxischen Effekte beobachtet. Der NOAEL für entwicklungstoxische Effekte liegt bei $500 \mathrm{mg} / \mathrm{kg} \mathrm{KG/d}$, der NOAEL für maternaltoxische Effekte bei 50 mg/kg KG/d.

In einer 1-Generationenstudie wurden neben der Entwicklungstoxizität auch Effekte auf das Immunsystem untersucht [126]. Hierzu wurde trächtigen
Ratten (Sprague-Dawley, $n=20$ ) während Tag 10 der Trächtigkeit bis Tag 20 nach der Geburt (PND 20) täglich Futter mit Konzentrationen von 0, 100, 1000, 10.000 ppm HBCDD verabreicht. Die interne Dosis wird mit 8-21, 81-213 und 803-2231 mg/kg KG/d angegeben. Zum Zeitpunkt PND20 und PNW11 wurden Nachkommen untersucht, die Muttertiere nur zu PND20. Bei den Muttertieren war in der höchsten Dosisgruppe das rel. Schilddrüsengewicht erhöht und es wurde histopathologisch eine erhöhte Anzahl und ein erhöhter Schweregrad einer diffusen Hypertrophie der Follikelzellen beobachtet. Der Fertilitätsindex sowie weitere Endpunkte zur Entwicklung waren bei den Nachkommen nicht beeinflusst. Zum Zeitpunkt PND20 war das Körpergewicht der weiblichen Nachkommen in der hohen Dosisgruppe reduziert (9\%). Das relative Lebergewicht war bei beiden Geschlechtern erhöht (27-28\%). Das Schilddrüsengewicht war in der mittleren und hohen Dosisgruppe zu beiden Zeitpunkten erhöht (signifikant bei Männchen, bei Weibchen nicht signifikant wegen eines erhöhten Mittelwertes mit hoher Standardabweichung). Darüber hinaus war die Schilddrüsenhormon-Konzentration von T3 im Serum verringert, und von TSH im Serum erhöht. Der NOAEL wird mit $100 \mathrm{ppm}(8-21 \mathrm{mg} / \mathrm{kg} \mathrm{KG/d})$ angegeben. Mit immunhistochemischen Me- thoden konnte ab Konzentrationen von 1000 ppm ein erhöhter Anteil an Vimentin(+), unreifen Astrozyten und Ret Protoonkogen $($ Ret $)(+)$ Oligodendrozyten in der Weißen Substanz des ZNS ermittelt werden [130], die mit einer Hypothyreose während der Entwicklung in Verbindung gebracht werden.

In einer ähnlichen Studie wurde der Einfluss auf die Entwicklung des Immunsystems untersucht [127]. Hierzu wurde trächtigen Ratten (Sprague-Dawley, $n=20)$ während Tag 10 der Trächtigkeit bis Tag 21 nach der Geburt (Postnatalwoche 3, PNW3) täglich Futter mit Konzentrationen von $0,100,1000,10.000$ ppm HBCDD verabreicht. Zum Zeitpunkt PNW3 und PNW11 wurden bei männlichen Nachkommen die Lymphozyten in der Milz, im Thymus sowie im peripheren Blut mittels Durchflusszytometrie auf die Expression von Oberflächenmarkern (CD3, CD4, CD8a, CD25, CD45RA, CD71 und CD161 (NKRP1A)) untersucht. Das Gewicht von Milz und Thymus sowie die Anzahl von weißen Blutkörperchen in diesen Organen waren unbeeinflusst. Das Schilddrüsenhormon T3 war zu beiden Zeitpunkten in der hohen Dosisgruppe signifikant verringert (in der mittleren Dosisgruppe nur zum Zeitpunkt PNW11). Die Konzentration von Serumalbumin war in der hohen Dosisgruppe erhöht. Die Durchflusszytometrie 
ergab nur geringe Unterschiede zum Zeitpunkt PNW3, die Effekte waren jedoch reversibel, zum Zeitpunkt PNW11 zeigten sich keine Unterschiede mehr.

Eriksson et al. [128] untersuchten die Effekte von HBCDD auf die Entwicklung des Nervensystems. Sie verabreichten 10 männlichen Mäusen (MNMRI) einmalig zum Zeitpunkt PND10 eine Dosis von 0; 0,9 bzw. 13,5 mg/kg KG. Im Alter von 3 Monaten zeigten sich Effekte auf Spontanbewegung und Lernfähigkeit bereits in der niedrigsten Dosisgruppe. Der LOAEL liegt bei $0,9 \mathrm{mg} / \mathrm{kg} \mathrm{KG}$.

\section{Bewertung}

\section{1 Übersicht über vorliegende Bewertungen}

Auf Basis der vorliegenden Studien für HBCDD wurden von verschiedenen Institutionen unterschiedliche NOAEL-Werte für die weitere Bewertung ausgewählt. Die vorliegenden Bewertungen sind in

- Tab. 11 zusammengefasst. In den meisten Fällen wurde kein TDI-Wert abgeleitet, sondern die Bewertung erfolgte über den Margin of Exposure (MoE). Im Rahmen der Bewertung von HBCDD als SVHC-Stoff durch die ECHA [6] wurden DNEL-Werte mit Sicherheitsfaktoren von 40 und 100 für systemische und reproduktionstoxische Effekte abgeleitet. Zur von der EFSA [7] verwendeten Studie mit einem LOAEL von $0,9 \mathrm{mg} / \mathrm{kg} \mathrm{KG}$ [128] merken die Autoren der EU Altstoffbewertung [1] an, dass diese Effekte nicht für eine Bewertung herangezogen werden sollten, bevor sie nicht durch eine zweite Studie in einem anderen Labor bestätigt würden. In der Bewertung von Health Canada [2] wird ebenso wie in der von der EFSA auf den in der Eriksson-Studie [128] adressierten Endpunkt „Effekte auf Spontanbewegung sowie Lern- und Gedächtnisfähigkeit“ zurückgegriffen. Hier wird dieser Endpunkt für die Charakterisierung der Exposition von Kindern verwendet (,it was considered appropriate to characterize the magnitude of the margin between potential exposures to infants and children and the behavioural effect LOAEL of $\left.0,9 \mathrm{mg} / \mathrm{kg} b w^{\prime \prime}\right)$. ECHA [5] merkt an, dass die Studie nicht nach aktuellen Richtlinien durchgeführt wurde und dass die expert group of the Existing Substance Regulation Technical Committee on New and Existing Substances (ESR TC NES) sie auch nicht als robust ansieht.

\section{Ableitung von HBM-Werten}

Die HBM-Kommission hat in ihrem Grundsatzpapier [131] mögliche Ableitungswege für HBM-Werte skizziert und die Voraussetzungen zur Ableitung von HBM-Werten aus tierexperimentellen Studien formuliert.

\subsection{Voraussetzung 1:Vorliegen eines anerkannten TDI/ADI - Wertes oder einer geeigneten Schlüsselstudie zur Toxizität}

Für HBCDD liegt bislang kein international oder national anerkannter Wert für eine tolerable tägliche Aufnahmemenge (TDI) vor.

Die EFSA (European Food Safety Authority) identifizierte Effekte auf die Entwicklung des Nervensystems und das Verhalten als kritischen Endpunkt toxikologischer Untersuchungen und leitete hierfür eine $\mathrm{BMDL}_{10}$ von $0,93 \mathrm{mg} / \mathrm{kg} \mathrm{KG}$ ab. In Übereinstimmung mit dem EFSA Panel on Contaminants in the Food Chain [7] erachtet auch die HBM-Kommission die Studie von Eriksson et al. [128] als Schlüsselstudie und verwendet die hieraus resultierende $\mathrm{BMDL}_{10}$ von $0,93 \mathrm{mg} /$ $\mathrm{kg}$ KG als POD für die Ableitung eines HBM-Wertes.

\subsection{Voraussetzung 2: Analytik}

Eine spezifische Methode zur Analyse der Ausgangssubstanz HBCDD in Plasma ist vorhanden. Die Nachweisgrenze beträgt 0,1 $\mu \mathrm{g} / \mathrm{L}$ (persönl. Mitteilung Prof. Dr. G. Leng, Currenta).

\subsection{Voraussetzung 3: Kinetische Basisdaten}

Die zur Verfügung stehenden toxikokinetischen Daten zeigen, dass oral verabreichtes HBCDD leicht resorbiert wird und sich im Fettgewebe anreichert. Da sich die Eliminationskinetik für Nage- tiere von der des Menschen unterscheidet, können die mit Effekten assoziierten äußeren Dosen aus dem Tierversuch nicht direkt für die Risikobewertung beim Menschen herangezogen werden. Vielmehr stellt die innere Dosis oder Körperlast das geeignete Maß für einen direkten Vergleich der Effekte aufTier und Mensch dar. Aus der $\mathrm{BMDL}_{10}$ von 0,93 mg/kg KG nach einmaliger oraler Gabe leitet sich unter Berücksichtigung einer oralen $\mathrm{Re}$ sorption bei Nagetieren von $85 \%$ eine tolerable Körperlast von 0,79 mg/kg KG ab. Dieser Wert muss durch einen Faktor von 0,32 dividiert werden (Körperfettgehalt bei Frauen 32\%), um die tolerable innere Dosis auf das Lipid-Kompartiment zu beziehen. Es ergibt sich somit ein Wert von: 2,47 mg/kg Fett

\subsection{Ableitung der HBM-I-Werte und Vergleich mit der Exposition der Allgemeinbevölkerung}

Nach Beaufschlagung mit einem Gesamt-Assessment-Faktor von 8 (Toxikodynamik: Interspezies $=2,5$ und Intraspezies $=\sqrt{10}$ ) wird für HBCDD ein HBMI-Wert von $0,3 \mathrm{mg} / \mathrm{kg}$ Fett, entsprechend $0,3 \mu \mathrm{g} / \mathrm{g}$ Fett (oder 1,6 $\mu \mathrm{g} / \mathrm{l}$ Plasma) festgelegt.

Daten zur Exposition der Allgemeinbevölkerung liegen vor. Sie beziehen sich auf den Nachweis der Ausgangssubstanz im Fettanteil der Muttermilch oder des Blutplasmas/-serums.

Die HBCDD Konzentrationen im Fettgewebe der Allgemeinbevölkerung liegen zwischen $<0,08$ und $10 \mathrm{ng} / \mathrm{g}$ Fett (Median) und damit deutlich unter dem HBM-I-Wert.

Danksagung. Die HBM-Kommission dankt Dr. Oliver Licht und Dr. Inge Mangelsdorf (Hannover) für die Erstellung des Textentwurfes, Dr. Birger Heinzow (Kiel), Petra Apel (Berlin), Prof. Dr. Hermann Fromme (München) und Prof. Dr. Jürgen Angerer (Bochum) für die ergänzenden Beiträge sowie Angela Lehmann (Berlin) für die Unterstützung bei der redaktionellen Bearbeitung.

\section{Einhaltung ethischer Richtlinien}

Interessenkonflikt. Die Autoren geben an, dass kein Interessenkonflikt besteht. 


\section{Bekanntmachungen - Amtliche Mitteilungen}

\section{Anhang}

Factsheet: HBM-value for HBCDD

\begin{tabular}{|c|c|c|c|}
\hline Substance & 1,2,5,6,9,10-Hexabromocyclodode & & \\
\hline Parameter & Value/Descriptor & Dimension & Comments \\
\hline HBM Guide value & & & \\
\hline Guide value II (Health hazard value) & n. a. & & \\
\hline Guide value I (Precautionary value) & 0,3 & $\mu \mathrm{g} / \mathrm{g}$ lipid & $\begin{array}{l}\text { Plasma/serum and breast milk lipid, adipose } \\
\text { tissue }\end{array}$ \\
\hline Year of issue & 2015 & & \\
\hline Status & Final & & 49. session of the HBM-commission \\
\hline General Information & & & \\
\hline CAS No & $25637-99-4$ & & $\begin{array}{l}3194-55-6 \text { (unspec.) 25637-99-4 (isomere } \\
\text { mixture) } \\
134237-50-6 \text { ( } a-H B C D D) \\
134237-51-7(\beta-H B C D D) \\
134237-52-8(\gamma-H B C D D)\end{array}$ \\
\hline IUPAC name & Hexabromocyclododecane & & \\
\hline Molar mass & 641,73 & $\mathrm{~g} / \mathrm{mol}$ & $\mathrm{C}_{12} \mathrm{H}_{18} \mathrm{Br}_{6}$ \\
\hline HBM-parameter & Unchanged substance & & Concentration in lipid \\
\hline Database - Value used for HBM Derivatio & & & \\
\hline $\begin{array}{l}\text { Tolerable intake } \\
\text { (TDI, RfD, DNEL) }\end{array}$ & 0,1 & $\mathrm{mg} / \mathrm{kg} \mathrm{bw} / \mathrm{d}$ & $\begin{array}{l}\text { DNEL (ECHA), value given for further informa- } \\
\text { tion, not used by the HBM commission }\end{array}$ \\
\hline Key study/Author(s) (Year) & Eriksson et al. [128] & & \\
\hline Species & Mice & & \\
\hline Route/type of study & Oral & & Oral dose \\
\hline Study length & $90 d$ & & Single oral dose on postnatal day 10 \\
\hline Exposure & Single oral dose on postnatal day 10 & & \\
\hline Critical endpoint/effect & Neurotoxicity & & Developmental neurotoxic effects \\
\hline POD & BMDL & $\mathrm{mg} / \mathrm{kg} \mathrm{bw}$ & \\
\hline POD Value & 0,93 & $\mathrm{mg} / \mathrm{kg} \mathrm{bw}$ & BMDL10 \\
\hline Assessment factors for HBM-value deriva & & & \\
\hline Dose-response assessment factor & - & & \\
\hline Severity of effect & - & & \\
\hline $\begin{array}{l}\text { Adjusted exposure duration factor (time } \\
\text { scaling) }\end{array}$ & n. a. & & Oral study \\
\hline Adjusted study length factor & n. a. & & \\
\hline Route-to-route extrapolation factor & n. a. & & \\
\hline Interspecies factor & - & & Kinetic \\
\hline & 2,5 & & Dynamic \\
\hline Intraspecies factor & - & & Kinetic \\
\hline & 3,16 & & Dynamic \\
\hline Sensitive population factor & - & & Included in study design \\
\hline $\begin{array}{l}\text { Other adjustment factors Quality of whole } \\
\text { database }\end{array}$ & - & & Klimisch (reliable) \\
\hline Total assessment factor (TAF) & 8 & & \\
\hline Kinetik terms for HBM-value derivation & & & \\
\hline Absorption factor & 0,85 & & \\
\hline Absorbed dose (internal POD) & 0,79 & $\mathrm{mg} / \mathrm{kg} \mathrm{bw}$ & $0,93 \times 0,85$ \\
\hline Percentage of fatty tissue (women) & 32 & $\%$ & Lipid compartment (women) \\
\hline
\end{tabular}


Factsheet: HBM-value for HBCDD (Fortsetzung)

Result - HBM Value

\begin{tabular}{|llll|}
\hline Internal POD/TAF & 0,099 & $\mathrm{mg} / \mathrm{kg} \mathrm{bw}$ & $0,79 / 8 ;$ absorbed single dose \\
\hline $\begin{array}{l}\text { Kinetic extrapolation body burden (fatty } \\
\text { tissue, lipid) and calculated HBM values }\end{array}$ & $0,099 / 0,32=0,31$ & $\mathrm{mg} / \mathrm{kg}$ lipid & $\begin{array}{l}\text { Rounded guide value: } 0,3 \mu \mathrm{g} / \mathrm{g} \text { lipid equivalent } \\
\text { to } 1,6 \mu \mathrm{g} / \mathrm{l} \text { plasma }\end{array}$ \\
\hline
\end{tabular}

Rationale.

HBM-Commission deliberated the HBM value for HBCDD on the basis of a dossier prepared by the Fraunhofer Institute (FhG, O. Licht and I. Mangelsdorf) on behalf of the Federal Environment Agency.

ECHA has used a NOAEC of $10 \mathrm{mg} / \mathrm{kg}$ bw from the 2-generation oral feeding study by Ema et al. [121] and derived a DNEL of 0,1 mg/kg bw/d by using a default UF of 100 $(10 \times 10)$. Aylward and Hays [132] estimated lipid-adjusted tissue concentrations in the laboratory animals at this point of departure in the range of $120.000 \mathrm{ng} / \mathrm{g}$ lipid. A provisional BE value of approximately $10.000 \mathrm{ng} / \mathrm{g}$ lipid was estimated correspondingly.

EFSA [7] identified neurodevelopmental effects on behaviour as the critical endpoint, and derived a benchmark dose lower confidence limit for a benchmark response of $10 \%$ (BMDL10) of $0,93 \mathrm{mg} / \mathrm{kg}$ body weight.

In accordance with the EFSA Panel on Contaminants in the Food Chain [7] the HBM-Commission considered the study of Eriksson et al. [128] as key study and the resulting BMDL10 as POD for the derivation of the HBM value.

The available toxicokinetic data suggest that orally administered HBCDD is easily absorbed and accumulates in lipid tissue. Because elimination kinetics of HBCDD in rodents and humans differ, external dose levels of HBCDD associated with toxic effects in animals cannot be simply extrapolated for the risk assessment in humans. Instead, the internal dose or body burden provides a more appropriate dose metric for a direct comparison of effects in animals and humans. Based on the calculated BMDL10 value of $0,93 \mathrm{mg} / \mathrm{kg}$ bw as derived from the Eriksson et al. study using a single oral administration, and considering an oral absorption in rodents of $85 \%$, a body burden at the BMDL 10 of $0,79 \mathrm{mg} / \mathrm{kg}$ bw was derived.

Standard assessment factors (AFs) for differences in toxicodynamics (interspecies $=2,5$ and intraspecies $=\sqrt{10}$ ) were applied (total assessment factor (TAF) $=8$ ).

With reference to a proportion of $32 \%$ fatty tissue (women) a factor of 0,32 has to be applied to relate the internal dose to the lipid compartment of the body.

The rounded $\mathrm{HBM}$ value is set at $0,3 \mu \mathrm{g} / \mathrm{g}$ lipid.

Concentrations in adipose tissue of the general population range from $<0,08$ to $10 \mathrm{ng} / \mathrm{g}$ lipid (median), which is clearly below the HBM-I value.

\section{Literatur}

1. European Union (EU) (2008) Risk assessment hexabromocyclododecane, CAS-No.: 25637-99-4, EINECS No.: 247-148-4, Final Report May 2008. S 492. basierend auf Angaben des American Chemistry Council Brominated Flame Retardant Industry Panel ACCBFRIP. http://ec.gc.ca/ese-ees/ 7882C148-8AE4-4BA4-8555-668C49F91500/ HBCD\%20-\%20FSAR\%20-\%20EN.pdf, http:// ec.gc.ca/ese-ees/B7060ED8-57FF-45AF-AD96BB022481ABC1/PC\%20table\%20-\%20HBCD EN.pdf, http://www.ec.gc.ca/toxiques-toxics/Default.asp?lang=En-US\&n=98E80CC6$1 \& \mathrm{xml}=58 \mathrm{~F} 1 \mathrm{CC} 80-7565-49 \mathrm{EA}-\mathrm{BC} 75$ 1739EC20DF1A http://flameretardants.americanchemistry.com/FAQs/Safety-Regulations.html

2. Environment Canada (2011) PROPOSED RISK MANAGEMENT APPROACH for Hexabromocyclododecane (HBCD) Chemical Abstracts Service Registry Number (CAS RN): 3194-55-6. http:// www.ec.gc.ca/ese-ees/default.asp?lang $=$ EnUS\&n=5F5A32FB-1\#i12, Screening Assessment Reports of Hexabromocyclododecane, Chemical Abstracts Service Registry Number 3194-55-6

3. National Industrial Chemicals Notification and Assessment Scheme (NICNAS) (2012) Priority Existing Chemical Assessment Report Hexabromocyclododecane - June ISBN 978-1-74241715-8, Publications approval number: D0755. http://www.nicnas.gov.au/, http://www.nicnas. gov.au/communications/publications/annual-reporting/nicnas-annual-report-2012-13

4. Heeb NV, Schweizer WB, Kohler M, Gerecke AC (2005) Structure elucidation of hexabromocyclododecanes - a class of compounds with a complex stereochemistry. Chemosphere 61(1):65-73. (http://www.sciencedirect.com/science/article/ pii/S0045653505004224)
5. European Chemicals Agency (ECHA) (2010a) Committee for Risk Assessment RAC Annex 1 Background Document to the Opinion proposing harmonised classification and labelling at Community level of Hexabromocyclododecane (HBCDD). ECHA/RAC/CLH-O-0000001050-94-03/ A1, Hexabromocyclododecane (HBCDD), EC number: 247-148-4 and 221-695-9, CAS number: 25637-99-4 and 3194-55-6, Adopted 8 December, $S 70$

6. European Chemicals Agency (ECHA) (2010b) Annex XV dossier, PROPOSAL FOR IDENTIFICATION OF A SUBSTANCE AS A CMR CAT 1 OR 2, PBT, $\checkmark P v B$ OR A SUBSTANCE OF AN EQUIVALENT LEVEL OF CONCERN, Proposal for identification of Hexabromocyclododecane as a SVHC, Substance Name: Hexabromocyclododecane, EC Number: 247-148-4, CAS Number: 25637-99-4, S 61

7. European Food Safety Authority (EFSA) (2011) Panel on Contaminants in the Food Chain (CONTAM); Scientific Opinion on Hexabromocyclododecanes (HBCDDs) in Food. EFSA Journal 9(7):2296. doi:10.2903/j.efsa.2011.2296

8. Organisation for Economic Co-operation and Development (OECD) (2007) SIDS Initial Assessment Profile for Cas. No. 25637-99-4, 3194-556, Hexabromocyclododecane (HBCDD). SIAM 24, 19-20 April. http://webnet.oecd.org/Hpv/ UI/handler.axd?id=ea58ac11-e090-4b24-b281200ae351686c

9. Bundesanstalt für Arbeitsschutz und Arbeitsmedizin (BAuA) Ausschuss für Gefahrstoffe (AGS) (2001) (Begründung für Bewertung von Stoffeigenschaften: 1,2,5,6,9,10-Hexabromocylodecan (HBCD). http://www.baua.de/de/Themenvon-A-Z/Gefahrstoffe/TRGS/pdf/905/905-1-2-56-9-10-hexabromocylodecan.pdf? blob=publicationFile $\& v=3$
10. Nordic Council of Ministers (2008) Hexabromocyclododecane as a possible global POP. Tema Nord Copenhagen.520, S 91. ISBN 978-92893-1665-1. http://www.unece.org/fileadmin/ DAM/env/documents/2008/EB/EB/Norway\%20 HBCDD\%20dossier.pdf

11. Arnot J, McCarty L, Armitage J, Toose-Reid L, Wania F, Cousins I (2009) An evaluation of hexabromocyclododecane (HBCD) for Persistent Organic Pollutant (POP) properties and the potential for adverse effects in the environment. Report submitted to European Brominated Flame Retardant Industry Panel (EBFRIP) May 26, S 214. http:// www.unece.org/fileadmin/DAM/env/documents/2009/EB/wg5/wgsr45/Informal\%20docs/ An\%20evaluation\%20of\%20hexabromocyclododecane_Final\%20report.pdf

12. UNITED NATIONS (UN) (2010) Report of the Persistent Organic Pollutants Review Committee on the work of its sixth meeting. Addendum: Risk profile on hexabromocyclododecane. UNEP/ POPS/POPRC.6/13/Add.2 Persistent Organic Pollutants Review Committee Sixth meeting Geneva. 11-15 October. http://chm.pops.int/Convention/POPsReviewCommittee/POPRCMeetings/ POPRC6/POPRC6Documents/tabid/783/Default. aspx

13. Marvin $\mathrm{CH}$, Tomy GT, Armitage JM, Arnot JA, McCarty L, Covaci A, Palace V (2011) Hexabromocyclododecane: current understanding of chemistry, environmental fate and toxicology and implications for global management. Environ Sci Technol 45(20):8613-8623

14. Umweltbundesamt (UBA) (2013) Weltweites "Aus" für Flammschutzmittel HBCD, Presse-Information 23/2013. 08. Mai http://www.umweltbundesamt.de/presse/presseinformationen/ weltweites-aus-fuer-flammschutzmittel-hbcd 
15. Hogue C (2013) Global Ban For Flame Retardant: United Nations: countries agree to stop use of hexabromocyclododecane under Stockholm Convention. News of The Week, C\&E N 91(19):6. http://cen.acs.org/articles/91/i19/Global-BanFlame-Retardant.html

16. Umweltbundesamt (UBA) (2015) Hintergrundpapier Hexabromcyclododecan (HBCD). Antworten auf häufig gestellte Fragen. http://www. umweltbundesamt.de/sites/default/files/medien/378/publikationen/faq_hbcd_1.pdf

17. Morose G (2006) An Overview of Alternatives to Tetrabromobisphenol A (TBBPA) and Hexabromocyclododecane (HBCD). A Publication of the Lowell Center for Sustainable Production, University of Massachusetts, Lowell, MA. S 32. http:// www.chemicalspolicy.org/downloads/AternativestoTBBPAandHBCD.pdf

18. Weil ED, Levchik SV (2009) Flame Retardants for Plastics and Textiles Practical Applications. Hanser Gardner Publications, Cincinnati, OH. S 315. ISBN: 978-3-446-41652-9

19. Institute of Occupational Medicine and IOM Consulting Ltd (IOM)(2008) Data on manufacture, import, export, uses and releases of HBCD as well as information on potential alternatives to its use. IOM Consulting, supported by BRE, PFA and Entec under framework contract ECHA/2008/2 S 108

20. Harrad S, de Wit CA, Abdallah MAE, Bergh C, Björklund JA, Covaci A, Darnerud PO, de Boer J, Diamond M, Huber S, Leonards P, Mandalakis M, Ostman C, Haug LS, Thomsen C, Webster TF (2010) Indoor contamination with hexabromocyclododecanes, polybrominated diphenyl ethers, and perfluoroalkyl compounds: an important exposure pathway for people? Environ Sci Technol 44(9):3221-3231. (http://pubs.acs. org/doi/abs/10.1021/es903476t)

21. de Wit CA, Björklund JA, Thuresson K (2012) Tridecabrominated diphenyl ethers and hexabromocyclododecane in indoor air and dust from Stockholm microenvironments 2: indoor sources and human exposure. Environ Int 39(1):141-147. (http://www.sciencedirect.com/science/article/ pii/S0160412011002583)

22. Abdallah MAE, Harrad S, Covaci A (2008a) Hexabromocyclododecanes and tetrabromobisphenol-A in indoor air and dust in Birmingham, UK: implications for human exposure. Environ $\mathrm{Sci}$ Technol 42(18):6855-6861. (http://pubs.acs.org/ doi/abs/10.1021/es801110a)

23. Takigami H, Suzuki G, Hirai Y, Sakai S (2009a) Brominated flame retardants and other polyhalogenated compounds in indoor air and dust from two houses in Japan. Chemosphere 76(2):270277. (http://www.sciencedirect.com/science/article/pii/S0045653509002902)

24. Kalachova K, Hradkova P, Lankova D, Hajslova J, Pulkrabova J (2012) Occurrence of brominated flame retardants in household and car dust from the Czech Republic. Sci Total Environ 441:182193. (http://www.sciencedirect.com/science/article/pii/S0048969712012612)

25. Roosens $L$, Abdallah MAE, Harrad S, Neels H, Covaci $A$ (2009) Exposure to hexabromocyclododecanes (HBCDs) via dust ingestion, but not diet, correlates with concentrations in human serum: preliminary results. Environ Health Perspect 117(11):1707-1712. (http://ehp.niehs.nih. gov/0900869/)
26. Harrad S, Abdallah MAE, Covaci A (2009) Causes of variability in concentrations and diastereomer patterns of hexabromocyclododecanes in indoor dust. Environ Int 35(3):573-579. (http:// www.sciencedirect.com/science/article/pii/ S0160412008002213)

27. Kajiwara N, Sueoka M, Ohiwa T, Takigami H (2009) Determination of flame retardant hexabromocyclododecane diastereomers in textiles. Chemosphere 74(11):1485-1489. (http:// www.sciencedirect.com/science/article/pii/ S0045653508014483)

28. Abdallah MAE, Harrad S, Ibarra C, Diamond M, Melymuk L, Robson M, Covaci A (2008b) Hexabromocyclododecanes in indoor dust from Cana$\mathrm{da}$, the United Kingdom, and the United States. Environ Sci Technol 42(2):459-464. (http://pubs. acs.org/doi/abs/10.1021/es702378t)

29. Fromme H, Hilger B, Kopp E, Miserok M, Völkel W (2014) Polybrominated diphenyl ethers (PBDEs), hexabromocyclododecane (HBCD) and "novel" brominated flame retardants in house dust in Germany. Environ Int 64:61-68. (http://www.sciencedirect.com/science/article/ pii/S $0160412013002778 \#)$

30. Björklund JA, Sellström U, de Wit CA, Aune M, Lignell S, Darnerud PO (2012) Comparisons of polybrominated diphenyl ether and hexabromocyclododecane concentrations in dust collected with two sampling methods and matched breast milk samples. Indoor Air 22(4):279-288. (http:// onlinelibrary.wiley.com/doi/10.1111/j.16000668.2011.00765.x/abstract)

31. Abb M, Stahl B, Lorenz W (2011) Analysis of brominated flame retardants in house dust. Chemosphere 85(11):1657-1663. (http:// www.sciencedirect.com/science/article/ pii/S 0045653511006655)

32. D'Hollander W, Roosens L, Covaci A, Cornelis C, Reynders H, Campenhout KV, Voogt Pd, Bervoets $\mathrm{L}$ (2010) Brominated flame retardants and perfluorinated compounds in indoor dust from homes and offices in Flanders, Belgium. Chemosphere. 81(4):478-487. (http://www.sciencedirect. com/science/article/pii/S0045653510008246)

33. Santillo D, Johnston $P$ (2003) Playing with fire: the global threat presented by brominated flame retardants justifies urgent substitution. Environ Int 29(6):725-734. (http://www.sciencedirect. com/science/article/pii/S0160412003001156)

34. Van den Eede N, Dirtu AC, Ali N, Neels H, Covaci A (2012) Multi-residue method for the determination of brominated and organophosphate flame retardants in indoor dust. Talanta 89:292-300. (http://www.sciencedirect.com/science/article/ pii/S003991401101099X)

35. Leonards PEG, Santillo D, Brigden K, van der Veen I, von Hesselingen J, de Boer J, Johnston P (2001) Brominated flame retardants in office dust samples. The second international workshop on brominated flame retardants: BFR 2001. May 1416, Stockholm, Schweden, P35, 233-236. (http:// www.bfr2013.com/abstract_download/2001/ upload/BFR2001_del4.pdf)

36. Ni HG, Zeng H (2013) HBCD and TBBPA in particulate phase of indoor air in Shenzhen, China. Sci Total Environ 458-460:15-19. (http:// www.sciencedirect.com/science/article/pii/ S0048969713004245)
37. Ali N, Dirtu AC, Van den Eede N, Goosey E, Har$\operatorname{rad} \mathrm{S}$, Neels H, 't Mannetje A, Coakley J, Douwes J, Covaci A (2012) Occurrence of alternative flame retardants in indoor dust from New Zealand: indoor sources and human exposure assessment. Chemosphere 88(11):1276-1282. (http:// www.sciencedirect.com/science/article/pii/ S0045653512004833)

38. Takigami H, Suzuki G, Hirai Y, Ishikawa Y, Sunami M, Sakai S (2009b) Flame retardants in indoor dust and air of a hotel in Japan. Environ Int 35(4):688-693. (http://www.sciencedirect.com/ science/article/pii/S0160412008002559)

39. Sudaryanto A, Isobe T, Suzuki G, Setiawan IE, Ilyas M, Riyadi AS, Takahashi S, Tanabe S (2009) Characterization of Brominated Flame Retardants in House Dust and Their Role as Non-Dietary Source for Human in Indonesia. Interdisciplinary Studies on Environmental Chemistry - Environmental Research in Asia 2. S 133-141 http://www.terrapub.co.jp/onlineproceedings/ec/02/pdf/ERA15. pdf

40. Stapleton HM, Allen JG, Kelly SM, Konstantinov A, Klosterhaus S, Watkins D, McClean MD, Webster TF (2008) Alternate and new brominated flame retardants detected in U.S. house dust. Environ Sci Technol 42(18):6910-6916. (http://pubs.acs. org/doi/abs/10.1021/es801070p)

41. Stapleton HM, Alaee M, Letcher RJ, Baker JE (2004) Debromination of the flame retardant decabromodiphenyl ether by juvenile carp (Cyprinus carpio) following dietary exposure. Environ Sci Technol 38(1):112-119. (http://pubs.acs.org/ doi/abs/10.1021/es034746j)

42. Fängström $B$, Athanassiadis $L$, Odsjö T, Norén K, Bergman A (2008) Temporal trends of polybrominated diphenyl ethers and hexabromocyclododecane in milk from Stockholm mothers, 1980-2004. Mol Nutr Food Res 52(2):187193. (http://onlinelibrary.wiley.com/doi/10.1002/ mnfr.200700182/abstract)

43. Lignell S, Aune M, Darnerud PO, Cnattingius S, Glynn A (2009) Persistent organochlorine and organobromine compounds in mother's milk from Sweden 1996-2006: compound-specific temporal trends. Environ Res 109(6):760-767. (http:// www.sciencedirect.com/science/article/pii/ S0013935109000826)

44. Bergman $\AA$, Hovander $L$, Sundström $M$, Athanassiadis I, Athanasiadou M, Sällsten G, Bignert A, Nyberg E (2010) Collection and chemical analysis of environmental contaminants in Swedish mother's milk - results from 2008-2010 (in Swedish=Insamling och kemisk analys av miljöföroreningar i svensk modersmjölk Resultat från 2008-2010. Rapport till Naturvårdsverket 2010-11-24 Överenskommelse/Avtal Nr. 215 0707 samt 215 0709. S 20. (MG 1064)). Swedish EPA Report \# 215 0707/215 0709, 1-22. http:// www.imm.ki.se/Datavard/Rapporter/Resultatrapport\%20NV\%20proj\%20215\%200707\%20 samt\%20215\%200709.pdf, http://naturvardsverket.diva-portal.org/smash/get/diva2:710318/ FULLTEXT01.pdf

45. Kakimoto K, Akutsu K, Konishi Y, Tanaka Y (2008) Time trend of hexabromocyclododecane in the breast milk of Japanese women. Chemosphere 71(6):1110-1114. (http://www.sciencedirect. com/science/article/pii/S004565350701301X) 
46. Kakimoto K, Akutsu K, Konishi Y, Toriba A, Hayakawa K (2012) Hexabromocyclododecane levels in foodstuff and human breast milk in Osaka, Japan. Interdisciplinary studies on environmental chemistry - environmental pollution and ecotoxicology, Bd 6. S 253-259. http://www.terrapub. co.jp/onlineproceedings/ec/06/pdf/PR631.pdf

47. Carignan CC, Abdallah MAE, Wu N, Heiger-Bernays W, McClean MD, Harrad S, Webster TF (2012) Predictors of tetrabromobisphenol-A (TBBP-A) and hexabromocyclododecanes (HBCD) in milk from Boston mothers. Environ Sci Technol 46(21):12146-12153. (http://pubs.acs.org/doi/ pdf/10.1021/es302638d)

48. Antignac JP, Cariou R, Maume D, Marchand P, Monteau F, Zalko D, Berrebi A, Cravedi JP, Andre F, Le Bizec B (2008) Exposure assessment of fetus and newborn to brominated flame retardants in France: preliminary data. Mol Nutr Food Res 52(2):258-265. (http://onlinelibrary.wiley.com/ doi/10.1002/mnfr.200700077/abstract)

49. Colles A, Koppen G, Hanot V, Nelen V, Dewolf MC, Noël E, Malisch R, Kotz A, Kypke K, Biot P, Vinkx C, Schoeters G (2008) Fourth WHO-coordinated survey of human milk for persistent organic pollutants (POPs): Belgian results. Chemosphere 73(6):907-914. (http://www.sciencedirect.com/ science/article/pii/S0045653508008679)

50. Ryan J, Wainman BC, Schecter A, Moisey J, Kosarac I, Sun WF (2006a) Trends of the brominated flame retardants PBDEs and HBCD, in human milks from North America. Organohalogen Compd 68:778-781. (http://www.dioxin20xx. org/pdfs/2006/06-81.pdf)

51. Ryan J, Wainman B, Schecter A (2006b) Trends of brominated flame retardants, PBDEs and $H B C D$, in human milks from North America. Dioxin 2006: 26th International Symposium on Halogenated Persistent Organic Pollutants, Oslo (NO), August 21-25, 2006, zitiert nach Health Canada 2011. http://www.ec.gc.ca/lcpe-cepa/ default.asp?lang=En-US\&n=A27E7A60-1\&offset $=13 \&$ toc $=$ show

52. Zegers BN, Mets A, Van Bommel R, Minkenberg C, Hamers T, Kamstra JH, Pierce GJ, Boon JP (2005) Levels of hexabromocyclododecane in harbor porpoises and common dolphins from western European seas, with evidence for stereoisomer-specific biotransformation by cytochrome p450. Environ Sci Technol 39(7):20952100. (http://www.abdn.ac.uk/marfish/pdfs/Zegers2005.pdf)

53. Roosens L, Cornelis C, D'Hollander W, Bervoets $L$, Reynders H, Van Campenhout K, Van Den Heuvel R, Neels H, Covaci A (2010) Exposure of the Flemish population to brominated flame retardants: model and risk assessment. Environ Int 36(4):368-376. (http://www.sciencedirect.com/ science/article/pii/S0160412010000267)

54. Abdallah MAE, Harrad S (2011) Tetrabromobisphenol-A, hexabromocyclododecane and its degradation products in UK human milk: relationship to external exposure. Environ Int 37(2):443448. (http://www.sciencedirect.com/science/article/pii/S0160412010002382)

55. Croes K, Colles A, Koppen G, Govarts E, Bruckers L, Van de Mieroop E, Nelen V, Covaci A, Dirtu AC, Thomsen C, Haug LS, Becher G, Mampaey M, Schoeters G, Van Larebeke N, Baeyens W (2012) Persistent organic pollutants (POPs) in human milk: a biomonitoring study in rural areas of Flanders (Belgium). Chemosphere. 89(8):988-994. (http://www.sciencedirect.com/science/article/ pii/S0045653512008673)
56. Glynn A, Lignell S, Darnerud PO, Aune M, Halldin Ankarberg E, Bergdahl IA, Barregård L, Bensryd I (2011) Regional differences in levels of chlorinated and brominated pollutants in mother's milk from primiparous women in Sweden. Environ Int 37(1):71-79. (http://www.sciencedirect.com/science/article/pii/S0160412010001479)

57. Lignell S, Glyn A, Darnerud PO, Aune M (2008) Temporal trends of PBDEs in mother's milk from Uppsala county, Bd 70. Organohalogen Compounds, Sweden, S 2123-2126. (http://www.dioxin20xx.org/pdfs/2008/08-579.pdf)

58. Lopez D, Athanasiadou M, Athanassiadis I, Estrada LD, Diaz-Barriga F, Bergman A (2004) A preliminary study on PBDEs and HBCD in blood and milk from Mexican Women. Proceedings from the third international workshop on brominated flame retardants, 6-9 June, Toronto, Canada, 482-487

59. Thomsen C, Frøshaug M, Broadwell SL, Becher G, Eggesb M (2005) Levels of brominated flame retardants in milk from the Norwegian human milk study; HUMIS. Organohalogen Compd 67:509-512. (http://www.dioxin20xx.org/ pdfs/2005/05-541.pdf)

60. Thomsen C, Frøshaug M, Leknes H, Becher $G$ (2003) Brominated flame retardants in breast milk from Norway. Organohalogen Compd 64:33-36. (http://www.dioxin20xx.org/ pdfs/2003/03-527.pdf)

61. Polder A, Thomsen C, Lindström G, Løken KB, Skaare JU (2008a) Levels and temporal trends of chlorinated pesticides, polychlorinated biphenyls and brominated flame retardants in individual human breast milk samples from Northern and Southern Norway. Chemosphere 73(1):1423. (http://www.sciencedirect.com/science/article/pii/S0045653508007558)

62. Thomsen C, Stigum H, Frøshaug M, Broadwell SL, Becher G, Eggesbø M (2010) Determinants of brominated flame retardants in breast milk from a large scale Norwegian study. Environ Int 36(1):68-74. (http://www.sciencedirect.com/science/article/pii/S0160412009002086)

63. Eljarrat E, Guerra P, Martinez E, Farre M, Alvarez JG, Lopez-Teijon M, Barcelo D (2009) Hexabromocyclododecane in human breast milk: levels and enantiomeric patterns. Environ Sci Technol 43(6):1940-1946. (http://pubs.acs.org/doi/ abs/10.1021/es802919e)

64. Schecter A, Harris TR, Shah N, Musumba A, Päpke $O$ (2008) Brominated flame retardants in US food. Mol Nutr Food Res 52(2):266-272. (http://onlinelibrary.wiley.com/doi/10.1002/mnfr.200700166/ abstract;jsessionid=A0107BD8F8F97898A6ABBA4D331F8192.f03t03)

65. Ryan J, van Oostdam J, Tittlemier S (Hrsg) (2005) Hexabromocyclododecane (HBCD) Presence in humans from Northern Regions, Northern Contaminants Program Results Workshop September 27-29, Victoria (BC), unpublished, zitiert nach Health Canada 2011

66. Malarvannan G, Kunisue T, Isobe T, Sudaryanto A, Takahashi S, Prudente M, Subramanian A, Tanabe $S$ (2009) Organohalogen compounds in human breast milk from mothers living in Payatas and Malate, the Philippines: levels, accumulation kinetics and infant health risk. Environ Pollut 157(6):1924-1932. (http://www.sciencedirect. com/science/article/pii/S0269749109000244)
67. Shi ZX, Wu YN, Li JG, Zhao YF, Feng JF (2009) Dietary exposure assessment of Chinese adults and nursing infants to tetrabromobisphenol-A and hexabromocyclododecanes: occurrence measurements in foods and human milk. Environ Sci Technol 43(12):4314-4319. (http://pubs.acs.org/ doi/abs/10.1021/es8035626)

68. Tue NM, Sudaryanto A, Minh TB, Isobe T, Takahashi S, Viet PH, Tanabe S (2010) Accumulation of polychlorinated biphenyls and brominated flame retardants in breast milk from women living in Vietnamese e-waste recycling sites. Sci Total Environ 408(9):2155-2162. (http:// www.sciencedirect.com/science/article/pii/ S0048969710000148)

69. Polder A, Gabrielsen GW, Odland JØ, Savinova TN, Tkachev A, Løken KB, Skaare JU (2008b) Spatial and temporal changes of chlorinated pesticides, PCBs, dioxins (PCDDs/PCDFs) and brominated flame retardants in human breast milk from Northern Russia. Sci Total Environ 391(1):41-54. (http://www.sciencedirect.com/science/article/ pii/S0048969707011114)

70. Toms LML, Guerra P, Eljarrat E, Barceló D, Harden F, Hobson P, Sjödin A, Ryan E, Mueller JF (2012) Brominated flame retardants in the Australian population: 1993-2009. Chemosphere 89(4):398-403. (http://www.sciencedirect.com/ science/article/pii/S0045653512006741)

71. Asante KA, Adu-Kumi S, Nakahiro K, Takahashi S, Isobe T, Sudaryanto A, Devanathan G, Clarke E, Ansa-Asare OD, Dapaah-Siakwan S, Tanabe $S$ (2011) Human exposure to PCBs, PBDEs and $H B C D$ s in Ghana: temporal variation, sources of exposure and estimation of daily intakes by infants. Environ Int 37(5):921-928. (http:// www.sciencedirect.com/science/article/pii/ S0160412011000626)

72. Thomsen C, Molander P, Daae HL, Janák K, Froshaug M, Liane VH, Thorud S, Becher G, Dybing E (2007) Occupational exposure to hexabromocyclododecane at an industrial plant. Environ Sci Technol 41(15):5210-5216. (http://pubs.acs.org/ doi/abs/10.1021/es0702622)

73. Kim U, Hong Y, Lee D, Sim W, Oh J (2013) PBDEs, HBCDs, TBBPA in infant-mother paired serum: focusing on investigating impact on thyroid hormone, relative proportion and relationship with environmental factors. POSTER SESSION: Toxicology, Epidemiology, Exposure Pathways, Policy P55. BFR2013 Sixth International Symposium On Flame Retardants April 7-10, San Francisco, California. http://www.bfr2013.com/upload/abstract-download/2013//Epi/1919_Un-Jung.pdf, http://www.bfr2013.com/

74. Fromme H, LGL (Bayerisches Landesamt für Gesundheit und Lebensmittelsicherheit) (2014) Persönliche Mitteilung zu den Ergebnissen einer Pilotuntersuchung

75. Odland JØ, Heimstad ES, Sandanger TM (2005) Kartlegging av miljøgifter i humane blodprøver fra Taimyr, Russland og Bodø, Norge - en pilotstudie av nye miljøgifter.Overvåkingsrapport Nummer: TA-2103, 930/05,30p, ISBN:82-7655-261-7. http://www.miljodirektoratet.no/old/klif/publikasjoner/overvaking/2103/2103.pdf

76. Odland JØ, Heimstad ES, Sandanger TM (2006) Kartlegging av „nye“ miljøgifter i humane blodprøver fra Nord-Norge, Nord-Vest Russland og Sibir. SPFO rapport: 963/2006, 28p. Statens forurensningstilsyn, Oslo, Norway, ISBN 82-7655290-0. http://www.miljodirektoratet.no/old/klif/ publikasjoner/overvaking/2184/ta2184.pdf 
77. Weiss J, Wallin $E$, Axmon A, Jonsson BAG, Åkesson H, Janák K, Hagmar L, Bergman A (2006) Hydroxy-PCBs, PBDEs, and HBCDs in serum from an elderly population of Swedish fishermen's wives and associations with bone density. Environ Sci Technol 40(20):6282-6289. (http://pubs.acs.org/ doi/abs/10.1021/es0610941)

78. Thomsen C, Knutsen HK, Liane VH, Frøshaug M, Kvalem HE, Haugen M, Meltzer HM, Alexander J, Becher $G$ (2008) Consumption of fish from a contaminated lake strongly affects the concentrations of polybrominated diphenyl ethers and hexabromocyclododecane in serum. Mol Nutr Food Res 52(2):228-237. (http://onlinelibrary.wiley. com/doi/10.1002/mnfr.200700123/abstract;jsessionid=1E81F255EC56881A7782999FED0F5B57. f02t04)

79. Peters RJB (2004) Man-made chemicals in human blood. TNO Report R2004/493. Prepared for Greenpeace Nederland, Nov 2004, 48p. http:// www.greenpeace.org/eu-unit/Global/eu-unit/reports-briefings/2009/3/man-made-chemicals-inhuman-bl.pdf

80. Meijer L, Weiss J, Van Velzen M, Brouwer A, Bergman A, Sauer PJ (2008) Serum concentrations of neutral and phenolic organohalogens in pregnant women and some of their infants in The Netherlands. Environ Sci Technol 42(9):34283433. (http://pubs.acs.org/doi/abs/10.1021/ es702446p)

81. Weiss J, Meijer L, Sauer P, Linderholm L, Athanasiadis I, Bergman Ã (2004) PBDE and HBCD levels in blood from Dutch mothers and infants. Analysis of a Dutch Groningen infant cohort. Organohalogen Compd 66:2677-2682. (http://www.dioxin20xx.org/pdfs/2004/04-328.pdf)

82. Pulkrabová J, Hrádková $\mathrm{P}, \mathrm{Haj}$ slová J, Poustka J, Nápravníková M, Polácek V (2009) Brominated flame retardants and other organochlorine pollutants in human adipose tissue samples from the Czech Republic. Environ Int 35(1):63-68. (http:// www.sciencedirect.com/science/article/pii/ S0160412008001542)

83. Johnson-Restrepo B, Adams DH, Kannan K (2008) Tetrabromobisphenol A (TBBPA) and hexabromocyclododecanes (HBCDs) in tissues of humans, dolphins, and sharks from the United States. Chemosphere 70(11):1935-1944. (http:// www.sciencedirect.com/science/article/pii/ S0045653507012374)

84. Goscinny S, Vandevijvere S, Maleki M, Van Overmeire I, Windal I, Hanot V, Blaude MN, Vleminckx C, Van Loco J (2011) Dietary intake of hexabromocyclododecane diastereoisomers ( $a-$ , $\beta$-, and $\gamma$-HBCD) in the Belgian adult population. Chemosphere 84(3):279-288. (http:// www.sciencedirect.com/science/article/pii/ S004565351100453X)

85. Törnkvist A, Glynn A, Aune M, Darnerud PO, Ankarberg EH (2011) PCDD/F, PCB, PBDE, HBCD and chlorinated pesticides in a Swedish market basket from 2005 - levels and dietary intake estimations. Chemosphere 83(2):193-199. (http:// www.sciencedirect.com/science/article/pii/ S0045653510014475)

86. Yu CC, Atallah YH (1980) Pharmacokinetics of $\mathrm{HBCD}$ in rats. Velsicol Chemical Corporation, 1-24. As cited in: European Commission (EU) 2007A. http://www.epa.gov/iris/pdfs/Litsearch_ HBCD.pdf
87. Arita R, Miyazaki K, Mure S (1983) Metabolic test of hexabromocyclododecane. Test on chemical substances used in household items. Studies on pharmacodynamics of hexabromocyclododecane. Department of Pharmacy, Hokkaido University Hospital (unpublished industry report) cited in EU RAR 2008

88. Ryuich A, Katsumi M, Shutoko M (1983) Test on chemical substances used in household items. Studies on pharmacodynamics of hexabromocyclododecane (unpublished paper translated into English). Department of Pharmacy, Hokkaido University Hospital

89. Commission on Life Sciences (2000) Hexabromocyclododecane. Toxicological risks of selected flame-retardant chemicals. National Research Council, National Academy Press, Washington DC, S 53-71. (http://www.ncbi.nlm.nih.gov/ books/NBK225647/, http://www.ncbi.nlm.nih. gov/books/n/nap9841/pdf/)

90. Van der Ven LT, Verhoef A, van de Kuil T, Slob W, Leonards PE, Visser TJ, Hamers T, Herlin M, Håkansson $\mathrm{H}$, Olausson $\mathrm{H}$, Piersma AH, Vos JG (2006) A 28-day oral dose toxicity study enhanced to detect endocrine effects of hexabromocyclododecane in Wistar rats. Toxicol Sci 94(2):281-292. (http://toxsci.oxfordjournals.org/ content/94/2/281.long)

91. Brandsma SH, Van der Ven LT, De Boer J, Leonards PE (2009) Identification of hydroxylated metabolites of hexabromocyclododecane in wildlife and 28-days exposed Wistar rats. Environ Sci Technol 43(15):6058-6063. (http://pubs.acs.org/doi/ pdf/10.1021/es900879k)

92. Szabo DT, Diliberto JJ, Hakk H, Huwe JK, Birnbaum LS (2010) Toxicokinetics of the flame retardant hexabromocyclododecane gamma: effect of dose, timing, route, repeated exposure, and metabolism. Toxicol Sci 117(2):282-293. (http:// toxsci.oxfordjournals.org/content/117/2/282. long)

93. Szabo DT, Diliberto JJ, Hakk H, Huwe JK, Birnbaum LS (2011a) Toxicokinetics of the flame retardant hexabromocyclododecane alpha: effect of dose, timing, route, repeated exposure, and metabolism. Toxicol Sci 121(2):234-244. (http:// toxsci.oxfordjournals.org/content/121/2/234. long)

94. Sanders JM, Knudsen GA, Birnbaum LS (2013) The Fate of $\beta$-Hexabromocyclododecane in Female C57BL/6 Mice. Toxicol Sci 134(2):251257. (http://toxsci.oxfordjournals.org/content/134/2/251.long)

95. Szabo DT, Diliberto JJ, Huwe JK, Birnbaum LS (2011b) Differences in tissue distribution of HBCD alpha and gamma between adult and developing mice. Toxicol Sci 123(1):256-263. (http:// toxsci.oxfordjournals.org/content/123/1/256. long)

96. Hakk H, Szabo DT, Huwe J, Diliberto J, Birnbaum LS (2012) Novel and distinct metabolites identified following a single oral dose of $a$ - or $\gamma^{-}$ hexabromocyclododecane in mice. Environ Sci Technol 46(24):13494-13503. (http://pubs.acs. org/doi/abs/10.1021/es303209g)

97. Germer S, Piersma AH, van der Ven L, Kamyschnikow A, Fery Y, Schmitz HJ, Schrenk D (2006) Subacute effects of the brominated flame retardants hexabromocyclododecane and tetrabromobisphenol A on hepatic cytochrome P450 levels in rats. Toxicology 218(2-3):229-236. (http:// www.sciencedirect.com/science/article/pii/ S0300483X05005342)
98. Esslinger S, Becker R, Maul R, Nehls I (2011) Hexabromocyclododecane enantiomers: microsomal degradation and patterns of hydroxylated metabolites. Environ Sci Technol 45(9):39383944. (http://pubs.acs.org/doi/abs/10.1021/ es1039584)

99. Huhtala S, Schultz E, Nakari T, Maclnnis G, Marvin C, Alaee M (2006) Analysis of hexabromocyclododecanes and their hydroxyl metabolites from in vitro and environmental samples by LC-MSMS. Organohalogen Cmpd 68:1987-1990. (http:// www.dioxin20xx.org/pdfs/2006/06-465.pdf)

100. Organisation for Economic Co-operation and Development (OECD) (2004) OECD Guidelines for the Testing of Chemicals, Section 4 Health Effects Test No. 428: Skin Absorption: In Vitro Method.1-8. ISBN: 9789264071087. http://www. oecd-ilibrary.org/environment/test-no-428-skinabsorption-in-vitro-method 9789264071087-en

101. Roper CS, Madden S, Biesemeier JA, Hoonagel H, Rothenbacker K (2007) The in vitro percutaneous absorption of radiolabelled hexabromocyclododecane (HBCD) through human skin. Organohalogen Compounds 69:2094-2095. (http://www. dioxin20xx.org/pdfs/2007/07-579.pdf)

102. Geyer HJ, Schramm KW, Darnerud PO, Aune M, Feicht EA, Fried KW, Henkelmann B, Lenoir D, Schmid P, McDonald TA (2004) Terminal elimination half-lives of the brominated flame retardants TBBPA, HBCD and lower brominated PBDEs in humans. Organohalogen Compounds 66:3820-3825. (http://www.dioxin20xx.org/ pdfs/2004/04-149.pdf)

103. Zeller H, Kirsch P (1969) Hexabromocyclododecane: 28-day Feeding Trials With Rats. BASF Institute for Industrial Hygiene and Pharmacology, Federal Republic of Germany. EPA/OTS Doc. \#86900000376 (not published)

104. Chengelis CP (1997) A 28-day repeated dose oral toxicity study of HBCD in rats, 1997, WIL Research Laboratories, Ashland, $\mathrm{OH}$, USA, S 925. (cited in EU RAR 2008)

105. Watanabe W, Shimizu T, Sawamura R, Hino A Konno K, Hirose A, Kurokawa M (2010) Effects of tetrabromobisphenol $A$, a brominated flame retardant, on the immune response to respiratory syncytial virus infection in mice. Int Immunopharmacol 10(4):393-397. (http:// www.sciencedirect.com/science/article/pii/ S1567576910000068)

106. Zeller H, Kirsch P (1970) Hexabromocyclododecane: 90-day Feeding Trials With Rats. Pharmakologisches Institute, BASF Institute for Industrial Hygiene and Pharmacology, Federal Republic of Germany. EPA/OTS Doc. \#86-900000380 (not published). (cited in EU RAR 2008)

107. Chengelis CP (2001) A 90-day oral (gavage) toxicity study of HBCD in rats, 2001, Wil Research Laboratories, Ashland, OH, USA, WIL-186012, $S$ 1527. (cited in EU RAR 2008)

108. Simmon V, Poole D, Newell G, Skinner W (1976) In vitro microbiological mutagenicity studies of four CIBA-GEIGY corporation compounds. Prepared for CIBA-GEIGY Corporation (not published); 5702, SRI Project LSC. (cited in EU RAR 2008)

109. Baskin AD, Phillips BM (1977) Mutagenicity of Two Lots of FM-100, Lot 53 and Residue of Lot 3322 in the Absence and Presence of Metabolic Activation. Industrial Bio-Test Laboratories, Northbrook, IL. EPA/OTS Doc. \#86-900000267. (cited in EU RAR 2008)

110. Gulf South Research Institute (GSRI) (1979) Mutagenicity test of GLS-S6-41A. (not published) 
111. Zeiger E, Anderson B, Haworth S, Lawlor T, Mortelmans K, Speck W (1987) Salmonella mutagenicity tests. III Results from the testing of 255 chemicals. Environ Mutagen 9(Suppl 9):1-60. (http://onlinelibrary.wiley.com/doi/10.1002/ em.2860090602/abstract)

112. Ogaswara S, Hanafusa T (1993) Report on mutagenicity test on pyroguard SR-103 using microorganisms(not published)

113. Hossack DJN, Richold M, Jones E, Bellamy RP (1978) Ames metabolic activation test to assess the potential mutagenic effect of compound no. 49.1-2. Huntingdon Research Centre UK. (cited in EU RAR 2008)

114. United States Environmental Protection Agency (EPA) 1990 Ames test with hexabromides. Letter from BASF. 86-900000379, EPA/OTS Doc. [cited in EU RAR 2008]. BASF (1990) Ames metabolic activation test to assess the potential mutagenic effect of und no. 49 With cover letter dated 031290. EPA/OTS Doc \#86-900000385; NTIS No. OTS0522948

115. Helleday $\mathrm{T}$, Tuominen $\mathrm{KL}$, Bergman $\AA$, Jenssen $\mathrm{D}$ (1999) Brominated flame retardants induce intragenic recombination in mammalian cells. Mutat Res 439(2):137-147. (http://www.sciencedirect. com/science/article/pii/S1383571898001867)

116. Organisation for Economic Co-operation and Development (OECD) (1997) OECD Guidelines for the Testing of Chemicals, Section 4 Health Effects Test No. 473: In Vitro Mammalian Chromosomal Aberration Test. 1-10. ISSN: 2074-5788. http://www.oecd.org/chemicalsafety/risk-assessment/1948434.pdf

117. Gudi R, Schadly EH (1996) Chromosome Aberrations in Human Peripheral Blood Lymphocytes. Microbiological Associates, Inc., Rockville, Md. Study No. G96A061.342. Sponsor: Chemical Manufacturers Association, Arlington, VA (not published)

118. Engelhardt, Hoffman (2000) Cytogenetic study in vivo with hexabromocyclododecane in the mouse micronucleus test after two intraperitoneal administrations. BASF, Ludwigshafen, Germany. (cited in EU RAR 2008)

119. Kurokawa Y, Inoue T, Uchida Y, Momma J (1984) Carcinogenesis test of flame retarder hexabromocyclododecane in mice (unpublished, translated into English). M. Hardy, Albermarle corporation, personal communication. Department of Toxicology. National Public Health Research Institute. Biological Safety Test and Research Centre. (cited in EU RAR 2008)

120. American Chemistry Council (ACC) (2001) HPV data summary and test plan for Hexabromocyclododecane (HBCD), CAS No. 3194556 Prepared by American Chemistry Council Brominated Flame Retardant Industry Panel (BFRIP) 1300 Wilson Blvd, Arlington, VA, December 20. http://www. epa.gov/chemrtk/pubs/summaries/cyclodod/ c13459.pdf

121. Ema M, Fujii S, Hirata-Koizumi M, Matsumoto M (2008) Two-generation reproductive toxicity study of the flame retardant hexabromocyclododecane in rats. Reprod Toxicol 25(3):335-351. (http://www.sciencedirect.com/science/article/ pii/S0890623807003383, http://www.ncbi.nlm nih.gov/pubmed/18262388)
122. Van der Ven $L T$, van de Kuil T, Leonards PE, Slob W, Lilienthal H, Litens S, Herlin M, Håkansson H, Cantón RF, van den Berg M, Visser TJ, van Loveren $\mathrm{H}$, Vos JG, Piersma AH (2009) Endocrine effects of hexabromocyclododecane (HBCD) in a one-generation reproduction study in Wistar rats. Toxicol Lett 185(1):51-62. (http://www.sciencedirect. com/science/article/pii/S037842740801360X)

123. Lilienthal $H$, van der Ven $L$, Piersma $A$, Vos J (2009) Effects of the brominated flame retardant hexabromocyclododecane (HBCD) on dopamine-dependent behaviour and brainstem auditory evoked potentials in a one-generation reproduction study in Wistar rats. Toxicol Lett 185(1):63-72. http://www.sciencedirect.com/science/article/pii/S0378427408013611

124. Murai T, Kawasaki H, Kanoh S (1985) Studies on the toxicity of insecticides and food additives in pregnant rats. Fetal toxicity of hexabromocyclododecane. Oyo Yakuri Pharmacometrics Japan 29(6):981-986. (cited in EU RAR 2008)

125. Stump DG (1999) A prenatal developmental toxicity study of hexabromocyclododecane (HBCD) in rats. WIL Research Laboratories Inc., Ashland, Ohio, laboratory study number WIL-186009, CMA reference number BFRIP 02 WIL-02 HBCD.1-410. EU RAR 2008

126. Saegusa Y, Fujimoto $H$, Woo GH, Inoue $K$, Takahashi M, Mitsumori K, Hirose M, Nishikawa A, Shibutani M (2009) Developmental toxicity of brominated flame retardants, tetrabromobisphenol $A$ and 1,2,5,6,9,10-hexabromocyclododecane, in rat offspring after maternal exposure from mid-gestation through lactation. Reprod Toxicol 28(4):456-467. (http://www.sciencedirect.com/ science/article/pii/S0890623809002044)

127. Hachisuka $A$, Nakamura $R$, Sato $Y$, Nakamura $R$, Shibutani M, Teshima R (2010) Effects of perinatal exposure to the brominated flame-retardant hexabromocyclododecane (HBCD) on the developing immune system in rats. Kokuritsu lyakuhin Shokuhin Eisei Kenkyusho Hokoku=Bulletin of National Institute of Health Sciences (128):5864 (auf Japanisch)

128. Eriksson P, Fischer C, Wallin $M$, Jakobsson E, Fredriksson A (2006) Impaired behaviour, learning and memory, in adult mice neonatally exposed to hexabromocyclododecane (HBCDD). Environ Toxicol Pharmacol 21(3):317-322. (http:// www.sciencedirect.com/science/article/pii/ S1382668905001821)

129. Organisation for Economic Co-operation and Development (OECD) (2001) OECD Guidelines for the Testing of Chemicals, Section 4 Health Effects Test No. 414: Prenatal Development Toxicity Study. 1-10. ISSN: 2074-5788

130. Fujimoto $H$, Woo GH, Morita R, Itahashi M, Akane H, Nishikawa A, Shibutani M (2013) Increased cellular distribution of vimentin and ret in the cingulum of rat offspring after developmental exposure to decabromodiphenyl ether or 1,2,5,6,9,10-hexabromocyclododecane. J Toxicol Pathol 26(2):119-129. (http://www.ncbi.nlm.nih gov/pmc/articles/PMC3695334/)

131. Kommission Human-Biomonitoring des Umweltbundesamtes (2014) Grundsatzpapier zur Ableitung von HBM-Werten. Stellungnahme der Kommission Human-Biomonitoring des Umweltbundesamtes. Bundesgesundheitsblatt Gesundheitsforschung Gesundheitsschutz 57(1):138-147. (http://link.springer.com/article/10.1007/s00103013-1867-2)
132. Aylward LL, Hays SM (2011) Biomonitoring-based risk assessment for hexabromocyclododecane (HBCD). Int J Hyg Environ Health 214(3):179187 